Proceedings of the 6th Polish Symposium of Physics in Economy and Social Sciences (FENS2012), Gdańsk, Poland

\title{
Almost Periodically Correlated Time Series in Business Fluctuations Analysis
}

\author{
Ł. $\operatorname{LENART}^{a, b, *}$ AND M. PIPIENं $\dot{1}^{a, c}$ \\ ${ }^{a}$ Economic Institute in National Bank of Poland, Ś więtokrzyska 11/21, 00-919 Warszawa, Poland \\ ${ }^{b}$ Department of Mathematics in Cracow University of Economics, Rakowicka 27, 31-510 Kraków, Poland \\ ${ }^{c}$ Department of Econometrics and Operations Research in Cracow University of Economics, Rakowicka 27, 31-510 \\ Kraków, Poland
}

\begin{abstract}
We propose a non-standard subsampling procedure to make formal statistical inference about the business cycle, one of the most important unobserved feature characterising fluctuations of economic growth. We show that some characteristics of business cycle can be modelled in a non-parametric way by discrete spectrum of the almost periodically correlated time series. On the basis of estimated characteristics of this spectrum business cycle is extracted by filtering. As an illustration we characterise the main properties of business cycles in industrial production index for Polish economy.
\end{abstract}

DOI: 10.12693/APhysPolA.123.567

PACS: 89.65.Gh, 05.10.Gg

\section{Introduction}

Seminal works, that originated interest in empirical modelling of business cycles in macroeconomy, clearly indicated their inhomogeneity for both, spatial and time domain. In particular, introductory remarks of Mitchell in Business Annals [1], contains the following suggestion: No two recurrences in all the array seem precisely alike. Business cycles differ in their duration as wholes and in the quickness and the uniformity with which they sweep from one country to another. When identifying business cycle Lucas proposed its own conception, which, as he states in his paper [2], identifies the business cycle with movements about trend in gross national income. These movements are typically irregular in period and in amplitude. Regularities are only observed in the co-movements among different aggregative time series; see [2]. It is clear that Mitchell initially suggested different time pattern of business cycles for different economies. However, it is obvious that from the dynamic point of view, as Lucas states, business cycle exhibits irregular and nonperiodic character.

For developed economies some stylised facts about business cycles are known in the literature; see [3] or [4]. But we see the lack of precise and well established methods of formal statistical modelling of those empirical properties. It prompts new studies resulting many different approaches and frameworks of business cycle extraction; see for example exhaustive review presented by Diebold and Rudenbush [5]. When the lack of the theory of statistical inference seems to be a persistent state, the consensus about empirical properties of business cy-

*corresponding author; e-mail: lukasz.lenart@uek.krakow.pl cles is based, either on an ad hoc reasoning, or on the empirical results, that are possible to confirm using a group of methods, built on the basis of relative different frameworks. However, the extraction of the business cycle component from observed time series is still a controversial issue. In particular, since there is ongoing interest in many approaches to separate growth component from the cyclical component, and because there is no consensus on how to detrend the data, the business cycle stylised facts are sensitive to the adopted procedure. Hence, this has become not only a controversial issue in the business cycle theory itself, but also a subject of criticism by competing empirical approaches, as well.

The main purpose of this paper is to present a novel approach to formal business cycle estimation. We propose a non-standard subsampling procedure, in order to make formal statistical inference about the properties of the business cycle. We show that business cycle can be modelled by parameters of discrete spectra of the almost periodically correlated (APC) stochastic process. The APC class is a generalisation of periodically correlated (PC) class of stochastic processes, introduced by Gladyshev [6]. The vast literature confirmed substantial empirical importance and flexibility of PC class in many time series applications, see [7-14]. According to Hurd and Miamee [15], the periodically correlated stochastic processes are nonstationary, but non-constant unconditional expectation of the process exhibits periodic, and hence regular, evolution in time domain. The generalisation presented in this paper assumes that the mean of the nonstationary time series can be described by almost periodic function, i.e. the function that belongs to the topological closure of periodic class of functions.

From the definition, APC stochastic processes may describe irregular character of unconditional means for nonstationary time series. Assuming that detrended time series follows APC, we relax assumption of stationarity of 
cyclical factor, very commonly imposed in filtering approaches. Nonstationarity of the cycle component of the series, together with possible irregularities in time pattern of the unconditional mean, makes our approach relatively flexible and general. Consequently, incorporating the APC factor into the model of observed discrete time series should result in much more accurate approach to business cycle extraction than those proposed so far.

In the empirical part of the paper we analyse the cyclical behaviour of production sector in Poland with the use of the model with APC stochastic component. We characterise business cycle on the basis of industrial production index and also on some subsector indices. We discuss the empirical results and reflect them to the previous analyses conducted for the Polish economy.

\section{Basic notation and definitions}

Formally, the second-order and real-valued time series $\left\{X_{t}: t \in \mathbb{Z}\right\}$ is called periodically correlated if both the mean function $\mu(t)=E\left(X_{t}\right)$ and the autocovariance function $B(t, \tau)=\operatorname{cov}\left(X_{t}, X_{t+\tau}\right)$ are periodic at $t$ for every $\tau \in \mathbb{Z}$, with the same period $T$. In order to introduce the class of almost periodically correlated time series we need the definition of almost periodic function. We recall the following definition from [16]:

Definition 2.1. A real-valued function $f(t): \mathbb{Z} \longrightarrow \mathbb{R}$ of an integer variable is called almost periodic ( $A P$ in short), if for any $\epsilon>0$ there exists an integer $L_{\epsilon}>0$, such that among any $L_{\epsilon}$ consecutive integers, there is an integer $p_{\epsilon}$ with the property

$$
\sup _{t \in \mathbb{Z}}\left|f\left(t+p_{\epsilon}\right)-f(t)\right|<\epsilon .
$$

A second-order real-valued time series $\left\{X_{t}: t \in \mathbb{Z}\right\}$ is called almost periodically correlated if both the mean function $\mu(t)=E\left(X_{t}\right)$ and the autocovariance function $B(t, \tau)=\operatorname{cov}\left(X_{t}, X_{t+\tau}\right)$ are almost periodic function of an integer variable, for every $\tau \in \mathbb{Z}$. It is easy to see that any periodic function is almost periodic. Therefore, the class of APC time series is wider than the class of PC time series. During last five decades the APC class was broadly applied in telecommunication ([17] and [18]), climatology [19] and many others fields. For exhaustive review of possible applications see [20] or [21]. Empirical importance of such a class of nonstationary time series prompted new studies concerning properties and estimation methods.

In APC case the mean function and the autocovariance function $B(t, \tau)$ for any $\tau \in \mathbb{Z}$ has the Fourier representation of the form

$$
\mu(t) \sim \sum_{\psi \in \Psi} m(\psi) \mathrm{e}^{\mathrm{i} \psi t}, \quad B(t, \tau) \sim \sum_{\lambda \in \Lambda_{\tau}} a(\lambda, \tau) \mathrm{e}^{\mathrm{i} \lambda t},
$$

where the Fourier coefficients $m(\psi)$ and $a(\lambda, \tau)$ are given by

$$
m(\psi)=\lim _{n \rightarrow \infty} \frac{1}{n} \sum_{t=1}^{n} \mu(t) \mathrm{e}^{-\mathrm{i} \psi t},
$$

$$
a(\lambda, \tau)=\lim _{n \rightarrow \infty} \frac{1}{n} \sum_{j=1}^{n} B(j, \tau) \mathrm{e}^{-\mathrm{i} \lambda j},
$$

see $[22,15]$. According to [16] sets $\Psi=\{\psi \in[0,2 \pi)$ : $\left.m_{X}(\psi) \neq 0\right\}$ and $\Lambda_{\tau}=\{\lambda \in[0,2 \pi): a(\lambda, \tau) \neq 0\}$ are countable. Hence, the set $\Lambda=\bigcup_{\tau \in \mathbb{Z}} \Lambda_{\tau}$ is also countable. If the time series is $\mathrm{PC}$, then representations (1) become equations and the sets $\Psi$ and $\Lambda$ are contained in the set $\{2 k \pi / T: k=0,1, \ldots, T-1\}$.

In the problem of business cycles extraction the vast econometric literature exploits approaches based on the assumption of zero mean imposed on the distribution of stochastic factor describing business fluctuations. Moreover, this stochastic factor is usually modelled under stationarity assumption, leading to the framework that utilises parameters of continuous spectrum. The econometric approach presented in this paper relaxes stationary assumption, and consequently a more general dynamic model of observed time series is subject to empirical analysis. We model business cycles in a non-parametric way, taking into account discrete spectra of observed time series. It means that we characterise business cycles by non-zero frequencies $\psi \in \Psi$ and by corresponding Fourier coefficients $m(\psi)$. The definition and properties of discrete spectra in simple representation see [23], or in $\mathrm{PC}$ case in [15].

Notice that any $\psi_{0} \in(0,2 \pi)$ corresponds to the length of the cycle $2 \pi / \psi_{0}$. Hence the following testing problem:

$$
H_{0}: \psi_{0} \notin \Psi, \quad H_{1}: \psi_{0} \in \Psi,
$$

enables to test the statistical significance of the cycle with appropriate length. According to the definition of the set $\Psi$ and Fourier coefficients $m(\cdot)$ our testing problem is equivalent to the following:

$$
H_{0}:\left|m\left(\psi_{0}\right)\right|=0, \quad H_{1}:\left|m\left(\psi_{0}\right)\right| \neq 0 .
$$

We consider formulation (4) in detail. Since we are interested in business cycle estimation we restrict frequency $\psi_{0}$ such that corresponding length of the cycle is not shorter than 1.5 years. This formally means that in further analysis for monthly data we assume that $\psi_{0} \in(0,0.35)$.

In this paper by amplitude, which corresponds to frequency $\psi \in \Psi \cap(0,0.35)$, we define the distance between maximum and minimum value of the function $h(t)=$ $2 \operatorname{Re}\left[m(\psi) \mathrm{e}^{\mathrm{i} \psi t}\right]$. The problem stated above requires statistical theory of detecting significant frequencies in the set $\Psi$ and corresponding Fourier coefficients $m(\cdot)$. In the next sections we present some results given sampling model generated by APC assumption.

\section{Estimation problem}

By $\left\{X_{c_{n}+1}, X_{c_{n}+2}, \ldots, X_{c_{n}+d_{n}}\right\}$ we denote a sample from APC time series $\left\{X_{t}: t \in \mathbb{Z}\right\}$, where $\left\{d_{n}\right\}_{n \in \mathbb{N}}$ is any sequence of integers tending to infinity with $n$ and $\left\{c_{n}\right\}_{n \in \mathbb{N}}$ is any sequence of integers. For any $\psi \in$ $[0,2 \pi)$, estimator $\hat{m}_{n}^{c_{n}, d_{n}}(\psi)\left(\hat{m}_{n}^{c, d}(\psi)\right.$ for short) of the parameter $m(\psi)$, in representation (1), based on sample $\left\{X_{c_{n}+1}, X_{c_{n}+2}, \ldots, X_{c_{n}+d_{n}}\right\}$ takes the form 


$$
\hat{m}_{n}^{c, d}(\psi)=\frac{1}{d_{n}} \sum_{j=c_{n}+1}^{c_{n}+d_{n}} X_{j} \mathrm{e}^{-\mathrm{i} \psi j} .
$$

The standardised version of (5) has asymptotic normal distribution with zero mean; see [24], Theorem 2.1. Additionally, the variance of this distribution is a function of values of the generalised spectral density, calculated at arguments dependent on $\psi$. For definition and basic properties of generalized spectral density in APC case see $[25,26]$. Since the standard theory, presented in [27], provides methods of estimation of generalised spectral density in APC case only under the zero-mean assumption or under the assumption that the set $\Psi$ is known and finite, estimation of generalised spectral density in our case is not possible so far. Therefore, in the paper we exploit subsampling methodology, to construct asymptotically consistent test related to (4). In this approach the asymptotic variance estimation is not of particular interest. Similarly, subsampling methodology was also used for PC case in time domain in [28] and for APC case in frequency domain in [27].

The problem of frequency estimation $\psi_{0}$ can be solved on the basis of a more generalised approach than presented by [29]. Given assumption that there exists interval $I_{\psi_{0}}$, such that $I_{\psi_{0}} \cap \Psi=\left\{\psi_{0}\right\}$, it is possible to formulate the natural estimator of the unknown frequency $\psi_{0}$ of the form $\hat{\psi}_{n}=\arg \max _{x \in I_{\psi_{0}}}\left(\sqrt{n}\left|\hat{m}_{n}(x)\right|\right)$. As it was shown in [24], Theorem 3.1, under some regularity conditions we have

$$
\left[\begin{array}{c}
\hat{m}_{n}^{c, d}\left(\hat{\psi}_{n}\right) \\
\hat{\psi}_{n}
\end{array}\right] \stackrel{p}{\longrightarrow}\left[\begin{array}{c}
m\left(\psi_{0}\right) \\
\psi_{0}
\end{array}\right] .
$$

\section{Subsampling procedure and consistency}

In this section we describe the main idea of the subsampling methodology, according to the approach presented and developed by Politis et al. [30]. We use the same notation. Initially we assume that the time series $\left\{X_{t}: t \in \mathbb{Z}\right\}$ is governed by unknown probability distribution $P$, that belongs to a certain class of probability measures $\mathcal{P}$. Denote by $\left\{X_{1}, X_{2}, \ldots, X_{n}\right\}$ a sample from the time series $\left\{X_{t}: t \in \mathbb{Z}\right\}$. Our goal is to approximate the distribution of

$$
v_{n}\left(\hat{\theta}_{n}-\theta(P)\right),
$$

where $\hat{\theta}_{n}=\hat{\theta}_{n}\left(X_{1}, X_{2}, \ldots, X_{n}\right)$ is an estimator of $\theta(P)$, the parameter of interests, and $v_{n}$ is appropriate normalising sequence. Let $b(n)$ ( $b$ for short) be any sequence of integer numbers tending to infinity with $n$, such that $b<n$ and $b / n \rightarrow 0$.

One of the main assumption in subsampling methodology is that there exists asymptotic distribution of (7). We denote this distribution by $J(P)$, with $J(x, P)$ as a corresponding cumulative distribution functions at point $x \in \mathbb{R}$. Following the idea of Politis et al. [30] the distribution of (7) can be approximated by its subsampling version of the form

$$
L_{n, b}(x)=\frac{1}{n-b+1} \sum_{t=1}^{n-b+11}\left\{v_{b}\left(\hat{\theta}_{n, b, t}-\hat{\theta}_{n}\right) \leq x\right\},
$$

where $\mathbf{1}\{B\}$ is the indicator function of the set $B$ and $\hat{\theta}_{n, b, t}=\hat{\theta}_{b}\left(X_{t}, X_{t+1}, \ldots, X_{t+b-1}\right)$ is an estimator of the unknown parameter $\theta(P)$ obtained on the basis of the sample $\left\{X_{t}, X_{t+1}, \ldots, X_{t+b-1}\right\}$, with $t$ as a starting point and $b$ as a size of subsample. Under suitable regularity conditions it is known that (see [30], Theorem 4.2.1, page 103):

(i) if $x$ is a continuity point of $J(\cdot, P)$, then $L_{n, b}(x) \stackrel{p}{\longrightarrow}$ $J(x, P)$,

(ii) if $J(\cdot, P)$ is continuous, then $\sup _{x \in \mathbb{R}} \mid L_{n, b}(x)-$ $J(x, P) \mid \stackrel{p}{\longrightarrow} 0$,

(iii) if $J(\cdot, P)$ is continuous at point $c(1-\alpha)$, then

$$
P\left(v_{n}\left(\hat{\theta}_{n}-\theta(P)\right) \leq c_{n, b}(1-\alpha)\right) \rightarrow 1-\alpha,
$$

where for any $\alpha \in(0,1)$, we define

$$
\begin{aligned}
& c_{n, b}(1-\alpha)=\inf \left(x: L_{n, b}(x) \geq 1-\alpha\right), \\
& c(1-\alpha)=\inf (x: J(x, P) \geq 1-\alpha) .
\end{aligned}
$$

The implication (iii) is crucial to construct a subsampling confidence interval for the parameter $\theta(P)$.

We are interested in estimation of the absolute value of coefficients of the Fourier representation of the mean of APC process. Namely, we take $\theta(P)=|m(\psi)|$. Subsampling procedure, with $\hat{\theta}_{n, b, t}=\left|m_{n}^{t-1, b}(\psi)\right|$ and $v_{n}=\sqrt{n}$, is consistent; see for details [24], Theorem 2.3. Consequently, the confidence intervals for the parameter $\theta(P)=|m(\psi)|$, obtained by subsampling procedure, are asymptotically consistent.

Now let us take any $\psi_{0} \in(0, \pi]$. The test (4) with test statistics $\Pi_{n}(\{\psi\})=\sqrt{n}\left|\hat{m}_{n}(\psi)\right|$ and subsampling critical value are asymptotically consistent. In our paper we prove some modification of this result (see Theorem (8.2) in Appendix). We use test statistics $\tilde{\Pi}_{n}(\{\psi\})=$ $\sqrt{n}\left|\hat{r}_{n}(\psi)\right|$, that can be interpreted as a value of test statistics $\Pi_{n}(\{\psi\})=\sqrt{n}\left|\hat{m}_{n}(\psi)\right|$ based on the sample $\left\{X_{1}-\overline{\boldsymbol{X}}_{n}, X_{2}-\overline{\boldsymbol{X}}_{n}, \ldots, X_{n}-\overline{\boldsymbol{X}}_{n}\right\}$, where $\overline{\boldsymbol{X}}_{n}$ is the sample mean for the path $\left\{X_{1}, X_{2}, \ldots, X_{n}\right\}$. The critical value $\tilde{c}_{n, b}^{\{\psi\}}(1-\alpha)$ is calculated according to the formula that utilises subsampling procedure

$$
\begin{aligned}
& \tilde{c}_{n, b}^{\{\psi\}}(1-\alpha)=\inf \left(x: \tilde{L}_{n, b}^{\{\psi\}}(x) \geq 1-\alpha\right), \\
& \tilde{L}_{n, b}^{\{\psi\}}(x)=\frac{1}{n-b+1} \\
& \quad \times \sum_{t=1}^{n-b+11}\left\{\sqrt{b}\left(\left|\hat{r}_{n}^{t-1, b}(\psi)\right|-\left|\hat{r}_{n}(\psi)\right|\right) \leq x\right\},
\end{aligned}
$$

where

$$
\hat{r}_{n}^{c, d}(\psi)=\frac{1}{d_{n}} \sum_{j=c_{n}+1}^{c_{n}+d_{n}}\left(X_{j}-\overline{\boldsymbol{X}}_{n}\right) \mathrm{e}^{-\mathrm{i} \psi j}
$$

and $\hat{r}_{n}(\psi)=\hat{r}_{n}^{0, n}(\psi)$.

\section{Statistical model of cyclical fluctuations}

In this section we present the statistical framework 
of extraction of the cyclical component when the one-dimensional time series describing changes in economic activity is observed. In Sect. 5.1 we present basic assumptions concerning the model, while in Sect. 5.2 we describe in detail the algorithm of formal statistical extraction of business cycle component.

\subsection{Model structure and assumptions}

Let consider a real-valued time series, denoted by $\left\{P_{t}\right.$ : $t \in \mathbb{Z}\}$. At the beginning of this section we assume that the unconditional expectation for the process $\left\{P_{t}: t \in \mathbb{Z}\right\}$ exists for any $t \in \mathbb{Z}$.

An interesting case that is of particular interest in econometrics is the class of integrated stochastic processes, denoted by $I(d)$ for integration of order $d \in \mathbb{N}$. If we are interested in analysis of $I(d)$ processes in our framework, some additional assumptions should be imposed top assumed the existence of unconditional moments. In the case when $\left\{P_{t}: t \in \mathbb{Z}\right\}$ is $I(1)$ process it is sufficient to assume additionally that there exists $t_{0} \in \mathbb{Z}$ such that $E\left(P_{t_{0}}\right)<\infty$. Hence we obtain in this case that $E\left(P_{t}\right)<\infty$ for any $t \in \mathbb{Z}$. More generally, if $\left\{P_{t}: t \in \mathbb{Z}\right\}$ follows $I(d)$ process, then it is sufficient to assume that there exists $t_{0} \in \mathbb{Z}$, such that $E\left(P_{t_{0}+k}\right)<\infty$ for $k=0,1, \ldots, d-1$, to assure moment existence. Consequently, we formally exclude in our analysis processes with pure integration, but some restricted cases, representing strict nonstationarity with finite unconditional mean, may be modelled.

For further analysis we assume that the mean function $\mu_{P}(t)=E\left(P_{t}\right)$ is defined by the sum of deterministic function $f(t, \beta)$, parameterized by $\beta \in \mathbb{R}^{p}$, and almost periodic function $g(t)$, with the Fourier expansion of the form

$$
g(t)=\sum_{\psi \in \Psi_{P}} m_{P}(\psi) \mathrm{e}^{\mathrm{i} \psi t} .
$$

For convenience, we rewrite $g(t)$ in equivalent representation

$$
g(t)=\sum_{\psi \in \Psi_{P} \cap[0, \pi]} a_{P}(\psi) \cos (\psi t)+b_{P}(\psi) \sin (\psi t) .
$$

This automatically implies that

$$
\mu_{P}(t)=f(t, \beta)+g(t)=f(t, \beta)+\sum_{\psi \in \Psi_{P}} m_{P}(\psi) \mathrm{e}^{\mathrm{i} \psi t}
$$

Equation (11) leads to a more general approach to modelling business fluctuations, than those presented in the literature so far; see for example [31-35]. The main advantage of our approach is that it only relies on the specification of the first moment of the time series $\left\{P_{t}: t \in \mathbb{Z}\right\}$, making model assumptions much weaker. To illustrate the importance of our assumptions and generalisation we present an example below.

Example 5.1. Let $\left\{P_{t}: t \in \mathbb{Z}\right\}$ be a time series such that $P_{t}=P_{t-1}+\epsilon_{t}$, where $E\left(P_{0}\right)=b$ and $\left\{\epsilon_{t}: t \in \mathbb{Z}\right\}$ is APC time series with expectation function $\mu_{\epsilon}(\cdot)$ such that $\mu_{\epsilon}(t)=a+g(t)-g(t-1)$, where $g: \mathbb{Z} \rightarrow \mathbb{R}$ is a function of the form

$$
g(t)=\sum_{\psi \in \Psi} m(\psi) \mathrm{e}^{\mathrm{i} \psi t},
$$

$a \in \mathbb{R}$ and $\operatorname{card}(\Psi)<\infty$. Notice that for any $t \geq 1$ we have

$$
P_{t}=P_{0}+\epsilon_{1}+\epsilon_{2}+\ldots+\epsilon_{t} .
$$

Therefore

$$
\begin{aligned}
& E\left(P_{t}\right)=b+\sum_{j=1}^{t} E\left(\epsilon_{j}\right) \\
& =b+a t-g(0)+g(t)=f(t, \beta)+g(t),
\end{aligned}
$$

where $f(t, \beta)=\beta_{0}+\beta_{1} t, \beta_{0}=b-g(0), \beta_{1}=a$. This means that time series $\left\{P_{t}: t \in \mathbb{Z}\right\}$ can be represented as (11). If $g(t) \equiv 0$, then $\mu_{\epsilon}(t)=a$, and time series $\left\{P_{t}: t \in \mathbb{Z}\right\}$ can be interpreted as $I(1)$ process with drift and assumption $E\left(P_{0}\right)=b$.

The function $f(t, \beta)$ can be interpreted as a trend component, modelled in this paper by the polynomial. The function $g(t)$ contains summarised information about seasonal fluctuations, business fluctuations and long-term cyclical fluctuations. From the Fourier representation of $g(t)$ we split the whole set $\Psi_{P}$ of non-zero frequencies into the mutually exclusive sets that are related to those three periodic attributes of time series dynamics. Initially, we interpret long-term cyclical fluctuations as those with the length more than 8 years, since the frequency $\omega$ is related to the length of cycle that equals $2 \pi / \omega$ units. In order to distinguish cyclical fluctuations from seasonal fluctuations we assume formally that in the representation (10) the set $\Psi_{P}=\{\psi: m(\psi) \neq 0\} \subset[0,2 \pi)$ is unknown. For the set $\Psi_{P}$, let us consider the following decomposition:

$$
\Psi_{P}=\Psi_{P, 1} \cup \Psi_{P, 2} \cup \Psi_{P, 3} .
$$

We assume that $\Psi_{P, 1} \cap(0,0.35)=\Psi_{P, 1}$, and consequently the set $\Psi_{P, 1}$ represents all frequencies with corresponding length of the cycle greater than 17 months. Therefore the set $\Psi_{P, 1}$ contains frequencies that can describe business fluctuations. The set $\Psi_{P, 2}$ contains only seasonal frequencies, namely $\Psi_{P, 2} \subset\{2 k \pi / 12: k=0,1, \ldots, 11\}$ while $\Psi_{P, 3}$ contains all remaining frequencies. In the following section we concentrate our attention only to parameter identification and estimation in the set $\Psi_{P, 1}$.

\subsection{Cycle identification and estimation}

Our approach aims at identification and estimation of cyclical fluctuations. In order to remove trend component and to weaken seasonal effects, observed time series is subject to some preliminary transformations. Hence, we formulate the algorithm of frequency identification that consists of three basic steps. The first step enables to remove seasonal component, the second step detects the trend component, while in the third step, parameter identification and estimation is provided.

Step 1 - removing the seasonal component. Seasonality appears in most monthly economic time series. More formally, we allow (it is assumed), that $\Psi_{P} \cap\{2 k \pi / 12: k=1,2, \ldots, 11\} \neq \emptyset$. Since, the esti- 
mation of the frequencies and corresponding Fourier coefficients that represent seasonal frequencies is not of particular importance in our paper, we use centered moving average filter $2 \times 12 \mathrm{MA}$ (see $[36,37]$ ) to remove seasonal pattern. We show below that this filter does not change the elements of the set $\Psi_{P, 1}$, which is crucial for future estimation procedure. Denote by $\left\{Y_{t}: t \in \mathbb{Z}\right\}$ time series obtained by application of the centered moving average filter. It means that $Y_{t}=L_{2 \times 12}(B) P_{t}$, where

$$
\begin{aligned}
& L_{2 \times 12}(B)=\left(B^{-6}+2 B^{-5}+\ldots+2 B^{-1}+2+2 B+\right. \\
& \left.\quad \ldots+2 B^{5}+B^{6}\right) / 24,
\end{aligned}
$$

and $B^{k} P_{t}=P_{t-k}$ for any $t$ and $k$. Let us note that the expectation of the time series $\left\{Y_{t}: t \in \mathbb{Z}\right\}$ exists. On the basis of Theorem 8.1 and elementary calculations we get

$$
\begin{aligned}
& \mu_{Y}(t)=E\left(Y_{t}\right)=\underbrace{\tilde{\beta}_{0}+\tilde{\beta}_{1} t+\ldots+\tilde{\beta}_{p} t^{p}}_{\tilde{f}(t, \tilde{\beta})} \\
& +\sum_{\psi \in \Psi_{Y}} m_{Y}(\psi) \mathrm{e}^{\mathrm{i} \psi t},
\end{aligned}
$$

where $\Psi_{Y} \cap\{2 k \pi / 12: k=1,2, \ldots, 11\}=\emptyset, \Psi_{Y}=$ $\Psi_{P} \backslash\{2 k \pi / 12: k=1,2, \ldots, 11\}$ and $\tilde{f}$ is a function. The Fourier coefficients $m_{P}(\psi)$ and $m_{Y}(\psi)$ are related according to the formula

$$
m_{Y}(\psi)=L_{2 \times 12}\left(\mathrm{e}^{-\mathrm{i} \psi}\right) m_{P}(\psi) .
$$

Notice that $\tilde{f}(t, \tilde{\beta})$ is also a polynomial of order $p$. In particular, for $s \in\{p-1, p\}$ we have $\tilde{\beta}_{s}=\beta_{s}$. Consequently, given model with $p=0$ or $p=1$ (i.e. constant or linear trend) we have that $\tilde{f}(t, \tilde{\beta}) \equiv f(t, \beta)$. In case $p=2$ functions $\tilde{f}$ and $f$ have different values, but $\tilde{f}(t, \tilde{\beta})-f(t, \beta)$ is constant over time. Additionally, filtering the series with centered moving average operator, we obtain that

$$
\Psi_{Y} \cap(0,0.35)=\Psi_{P, 1}
$$

and

$$
\Psi_{Y} \cap \Psi_{P, 2}=\emptyset,
$$

which means that the set $\Psi_{Y}$ still contains the same elements as $\Psi_{P, 1}$ and does not contain seasonal frequencies from the set $\Psi_{P, 2}$.

Step 2 - removing the trend component. The case when $p=0$ is trivial. Let consider the case $p=1$. Application of difference operator $L_{1}(B)=1-B$ for the time series $\left\{Y_{t}: t \in \mathbb{Z}\right\}$ results with time series $\left\{X_{t}: t \in\right.$ $\mathbb{Z}\}$ :

$$
\begin{aligned}
X_{t} & =L_{1}(B) Y_{t}=Y_{t}-Y_{t-1} \\
& =\left(P_{t+6}-P_{t-5}+P_{t+5}-P_{t-6}\right) / 24 .
\end{aligned}
$$

The expectation of the time series $\left\{X_{t}: t \in \mathbb{Z}\right\}$ exists and is described by almost periodic function of the form

$$
\mu_{X}(t)=\beta_{1}+\sum_{\psi \in \Psi_{X}} m_{X}(\psi) \mathrm{e}^{\mathrm{i} \psi t},
$$

where

$$
\Psi_{X} \subset\{0\} \cup \Psi_{P} \backslash\{2 k \pi / 12: k=1,2, \ldots, 11\},
$$
and

$$
\Psi_{X} \cap(0,0.35)=\Psi_{P, 1},
$$

which follows from Theorem 8.1. Additionally, we have

$$
\begin{aligned}
& m_{X}(\psi)=L_{1}\left(\mathrm{e}^{-\mathrm{i} \psi}\right) m_{Y}(\psi) \\
& \quad=L_{1}\left(\mathrm{e}^{-\mathrm{i} \psi}\right) L_{2 \times 12}\left(\mathrm{e}^{-\mathrm{i} \psi}\right) m_{P}(\psi)
\end{aligned}
$$

and Assumption 8.1 holds.

In the general case, when $p \in \mathbb{N}$ we use natural operator $L_{p}(B)=(1-B)^{p}$. The resulting time series $\left\{X_{t}: t \in \mathbb{Z}\right\}$ can be represented by the following transformation of $Y_{t}$ :

$$
X_{t}=(1-B)^{p} Y_{t},
$$

and hence, the expectation of $X_{t}$ takes the form

$$
E\left(X_{t}\right)=\mu_{X}(t)=p ! \beta_{p}+\sum_{\psi \in \Psi_{X}} m_{X}(\psi) \mathrm{e}^{\mathrm{i} \psi t}
$$

where, according to Theorem 8.1

$$
\begin{aligned}
& m_{X}(\psi)=L_{p}\left(\mathrm{e}^{-\mathrm{i} \psi}\right) m_{Y}(\psi) \\
& \quad=L_{p}\left(\mathrm{e}^{-\mathrm{i} \psi}\right) L_{2 \times 12}\left(\mathrm{e}^{-\mathrm{i} \psi}\right) m_{P}(\psi) .
\end{aligned}
$$

By estimation $\left|L_{p}\left(\mathrm{e}^{-\mathrm{i} \psi}\right) L_{2 \times 12}\left(\mathrm{e}^{-\mathrm{i} \psi}\right)\right|>0$, which is true for any $p \in \mathbb{N}$ and $\psi \in(0,0.35)$, we have

$$
\Psi_{X} \cap(0,0.35)=\Psi_{P, 1} \text {. }
$$

Therefore the problem of parameter identification and estimation in the set $\Psi_{P, 1}$ reduce to the problem of parameter identification and estimation in the set $\Psi_{X} \cap(0,0.35)$.

Step 3 - parameter identification and estimation. The formula (21) is crucial in the algorithm of parameter identification and estimation in the set $\Psi_{P, 1}$. Initially, in Step 3 we formulate the additional assumption that the autocovariace function of time series $\left\{X_{t}: t \in\right.$ $\mathbb{Z}\}$ exists and it is almost periodic function. Notice that the weaker assumption concerning periodic structure of autocovariance function appears in the literature concerning analysis of economic time series; see for example $[7-10,12,14]$. We use statistics $\tilde{\Pi}_{n}(\{\psi\})=\sqrt{n}\left|\hat{r}_{n}(\psi)\right|$ and corresponding critical value $\tilde{c}_{n, b}(0.99 \%)$ for the series generated from the previous steps of the algorithm. The test statistics $\tilde{\Pi}_{n}(\{\psi\})$ can be interpreted as a value of test statistics $\Pi_{n}(\{\psi\})=\sqrt{n}\left|\hat{m}_{n}(\psi)\right|$ based on the sample $\left\{X_{1}-\overline{\boldsymbol{X}}_{n}, X_{2}-\overline{\boldsymbol{X}}_{n}, \ldots, X_{n}-\overline{\boldsymbol{X}}_{n}\right\}$, where $\overline{\boldsymbol{X}}_{n}$ is the sample mean for the path $\left\{X_{1}, X_{2}, \ldots, X_{n}\right\}$. The critical value is calculated according to the formula that utilises subsampling procedure presented in [30]:

$$
\tilde{g}_{n, b}^{\{\psi\}}(1-\alpha)=\inf \left(x: \tilde{G}_{n, b}^{\{\psi\}}(x) \geq 1-\alpha\right),
$$

where

$$
\tilde{G}_{n, b}^{\{\psi\}}(x)=\frac{1}{n-b+1} \sum_{t=1}^{n-b+11}\left\{\sqrt{b}\left|\hat{r}_{n}^{t-1, b}(\psi)\right| \leq x\right\} .
$$

We fix $b=2.5 \sqrt{n}$ and we calculate test statistics and corresponding critical value for $\psi$ from the discrete set of frequencies on the interval $(0,0.35)$. If the value of test statistics is greater than the critical value on some subinterval $I \subset(0,0.35)$ we take this subinterval as the interval containing some elements of the set $\Psi_{P, 1}$. Next, we estimate the frequency connected with subinterval $I$ using (6). By plug in technique we estimate amplitude related to each identified frequency in almost periodic part of the mean function of the process $\left\{P_{t}: t \in \mathbb{Z}\right\}$.

In our algorithm step 3 is fundamental in procedure of 
extraction business cycle component from the observed time series. Its main advantage is that the frequencies describing the cyclical dynamics of economic activity are subject to formal statistical inference. This clearly distinguishes our approach from many other procedures presented in the literature, where the lack of statistical uncertainty in the procedure is very common and forces ad hoc approach; see for example polemics concerning detrending in [38] and [39].

However, it is very important that the procedure yields only statistically significant frequencies, and extraction of the business cycle is subject to additional filtering. In the empirical part of the paper we use the Hodrick-Prescott (HP) filter (see [40]), with smoothness parameter $\lambda$. According to $[41-43]$ parameter $\lambda$ can be described as the argument of frequency $\psi_{0}$ :

$$
\lambda=\frac{1}{4\left[1-\cos \left(\psi_{0}\right)\right]^{2}},
$$

where $2 \pi / \psi_{0}$ can be interpret as a length of the cycle. Hence, on the basis of our procedure, it is possible to choose appropriate parameter $\lambda$ of the HP filter, restricting spectrum only to significant parameters in the set $\Psi_{P, 1}$. Alternatively it is possible to apply any filter in cycle extraction. We choose the simplest HP filter for illustrative purposes.

\section{Empirical illustration}

In this part of the paper we analyse cyclical behaviour of production sector in Poland. In particular we apply our model and three step procedure in order to characterise business cycles in industrial production index and some subsector indices.
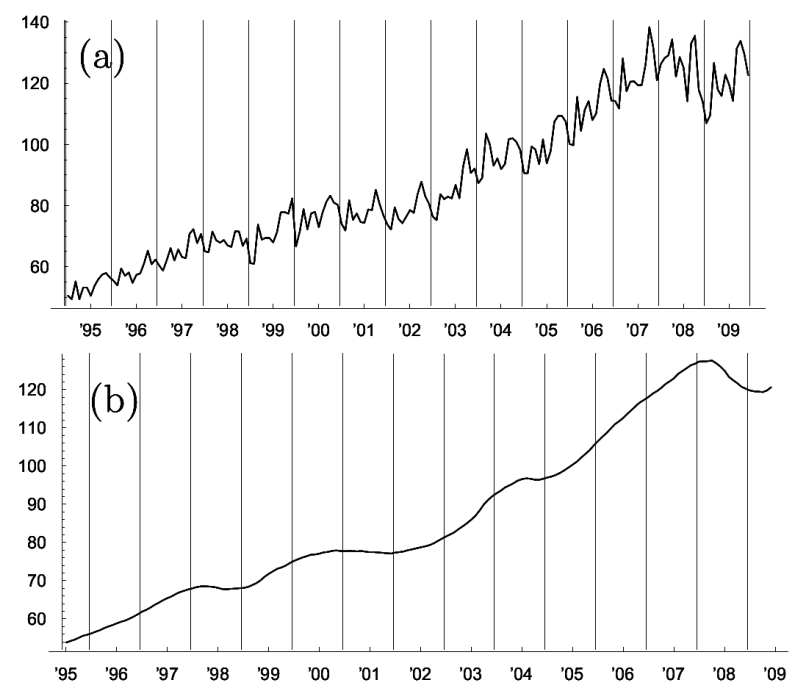

Fig. 1. (a) Industrial production index in Poland $(2005$ year $=100 \%)$ from January 1995 to December 2009; (b) realization of centered moving average filter $2 \times 12 \mathrm{MA}$ applied for industrial production index in Poland.

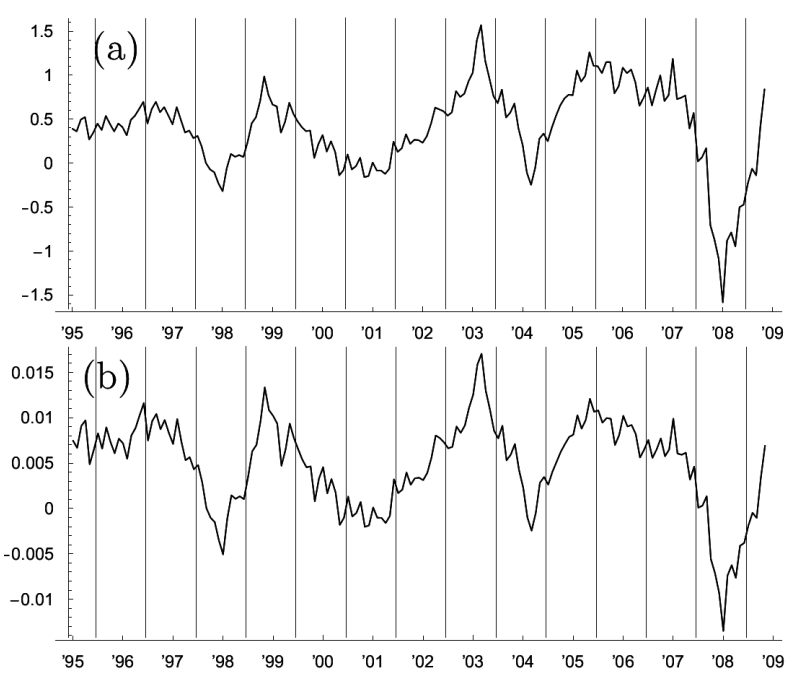

Fig. 2. (a) First difference of centered moving average filter $2 \times 12$ MA applied for industrial production index; (b) first difference of centered moving average filter $2 \times$ $12 \mathrm{MA}$ applied for logarithm of industrial production index.

Figure 1a presents time series of industrial production index ${ }^{\dagger}$ in Poland from January 1995 to December 2009 (2005 year $=100 \%$ ). This index contains: mining and quarrying; manufacturing; electricity, gas, steam and air conditioning supply. In the first step we applied centered moving average filter $2 \times 12 \mathrm{MA}$ to eliminate strong seasonal effects. The results of filtering is plotted in Fig. 1b. It is clear that centered moving average filter removes the seasonal effects and also business fluctuations are clearly observable (see Fig. 1b).

According to our algorithm, presented in previous sections, we present in Fig. 2a first differences of the centered moving average filter applied for industrial production index. We see some evidence about the existence of cyclical behavior in time series under consideration. The amplitude of cycle does not seem to be constant over time. Also, the amplitude is smaller in period 19952001, while after year 2001 is characterized by greater variability. Therefore we use logarithm transformation for industrial production index to stabilize the amplitude. Figure $2 \mathrm{~b}$ presents the first difference of centered moving average filter applied for logarithm of industrial production index. It is easy to see that the amplitude is more constant over time than before logarithm transformation.

Figure 3 presents plots of the values of the test statistics $\tilde{\Pi}_{n}(\{\psi\})=\sqrt{n}\left|\hat{r}_{n}(\psi)\right|$ with corresponding critical value $\tilde{c}_{n, b}(0.99 \%)$. The test statistics exceeds the critical value in three subsets on the interval $(0,0.35)$. Hence, taking care only of significant values of test statistic in Fig. 3 and in zoom in Fig. 4, we assume that

\footnotetext{
†Source: Eurostat.
} 


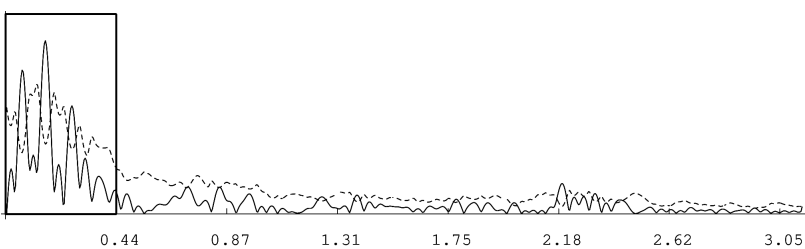

Fig. 3. Frequency identification using statistics $\tilde{\Pi}_{n}(\{\psi\})=\sqrt{n}\left|\hat{r}_{n}(\psi)\right|$ and corresponding critical value $\tilde{c}_{n, b}(0.99 \%)$ for the realization of time series $\left\{X_{t}: t \in \mathbb{Z}\right\}$ : continuous line - the value of test statistics $\tilde{\Pi}_{n}(\{\psi\})=\sqrt{n}\left|\hat{r}_{n}(\psi)\right|$ for $\psi$ from the set $\{(k-1) \pi / 720: k=1,2, \ldots, 720\}$; dashed line - critical value $\tilde{c}_{n, b}^{\{\psi\}}(99 \%)$ for $\psi$ from the set $\{(k-1) \pi / 720: k=1,2, \ldots, 720\}$.

$$
\Psi_{P, 1} \cap(0,0.35)=\left\{\psi_{1}, \psi_{2}, \psi_{3}\right\} .
$$

The values of estimated frequencies from the set $\Psi_{P, 1}$ were calculated according to (6). These values with corresponding length of the cycle can be found in Table I.

Estimated amplitude of the cycle with corresponding length 8.5 and 3.4 years equal 0.13 and 0.07 , respectively.
This second amplitude dominates the estimated value of amplitude with corresponding length 2 years. We see that 8.5-year length of the cycle received data support. However we cannot formally interpret such fluctuations as a long-term growth trend or business fluctuations. We should rather look at these fluctuations as a mixture of both long-term growth trend and business fluctuations. Consequently and unquestionable, the dataset support fluctuations with corresponding length 3.4 years as a basic characteristic of business cycle in industrial production in Poland. To confirm this statement we extract cyclical fluctuations from industrial production index (filtered by centered moving average filter $2 \times 12 \mathrm{MA}$ ) with the use of HP filter condition to the values of parameter $\lambda$ fixed for $\lambda=5500, \lambda=12000, \lambda=32000, \lambda=55000$. The results are plotted in Fig. 5. By restricting parameter $\lambda$ to values stated above, according to the formula 22, we extract fluctuations with the length not greater than $4.5,5.5,7$ and 8 years, respectively. Since our goal was to extract only business fluctuations without significant influence of long-term growth trend, we restrict filtering only to fluctuations with corresponding length shorter than 8 years.

Estimated frequencies with corresponding length of the cycle for industry production index in Poland.

\begin{tabular}{c|c|c|c}
\hline \hline The value of frequency estimator & $\hat{\psi}_{n, 1}=0.062$ & $\hat{\psi}_{n, 2}=0.153$ & $\hat{\psi}_{n, 3}=0.258$ \\
Corresponding length of the cycle (in years) & 8.5 & 3.4 & 2
\end{tabular}

TABLE II

Expansions and recessions in industrial production index in Poland in the period July 1995 - June 2009.

\begin{tabular}{c|c|c|c|c|c}
\hline \hline Expansion & ..-Dec. 97 & Feb. 99-May 00 & Sept. 02-Mar. 04 & Jun. 05-Jan. 08 & Apr. 09-... \\
Recession & Dec. 97-Feb. 99 & May 00-Sept. 02 & Mar. 04-Jun. 05 & Jan. 08-Apr. 09 &
\end{tabular}

Analysing plots presented in Fig. 5 it is possible to confirm the presence of cycles in industrial production in Poland with estimated length in the interval 3-4 years (during the period 1995-2009). In Table II we determined the periods of recessions and expansions in industrial production. We interpret turning points as margins of this periods. In most cases the recession is shorter than expansion. Consequently, our analysis confirms results discussed in the literature that business cycle in industrial production for Poland displays asymmetric behaviour. Also, the business cycle troughs are rather sharper than peaks, which is also typical for business cycles; see $[44,45]$.

In the next step we provide a more detailed analysis based on a formal identification of business cycles in sectors and subsectors of industrial production in Poland. We use the same statistical tools as for the total industrial production index. We considered all categories identified for industrial production. The set of all modelled indices are presented in Table III.

Figure 6 presents plots of logarithms of all considered indices. Repeating the procedure, initially applied for the total index, we use centered moving average filter $2 \times 12 \mathrm{MA}$ to remove seasonal effect from the data sets (see Fig. 7). First differences are presented in Fig. 8. It is clear that majority of indices exemplify cyclical pattern, just like in the case of index of total production, but with rather differential amplitudes and length. To identify frequencies in the unknown set $\Psi_{P, 1}$ we applied again the test statistic $\tilde{\Pi}_{n}(\{\psi\})=\sqrt{n}\left|\hat{r}_{n}(\psi)\right|$ and corresponding critical value $\tilde{c}_{n, b}^{\{\psi\}}(\alpha)$. The results are presented in Figs. 9 and 10, where we plotted estimated lengths of the cycles together with appropriate estimated amplitude. In different sectors and subsectors of industrial production the data provide evidence in favour of cycles with length in the interval 1.5-3 years. However, those cycles are characterized by much shorter estimated amplitude than the cycles with length in the interval 3-4 years. The 

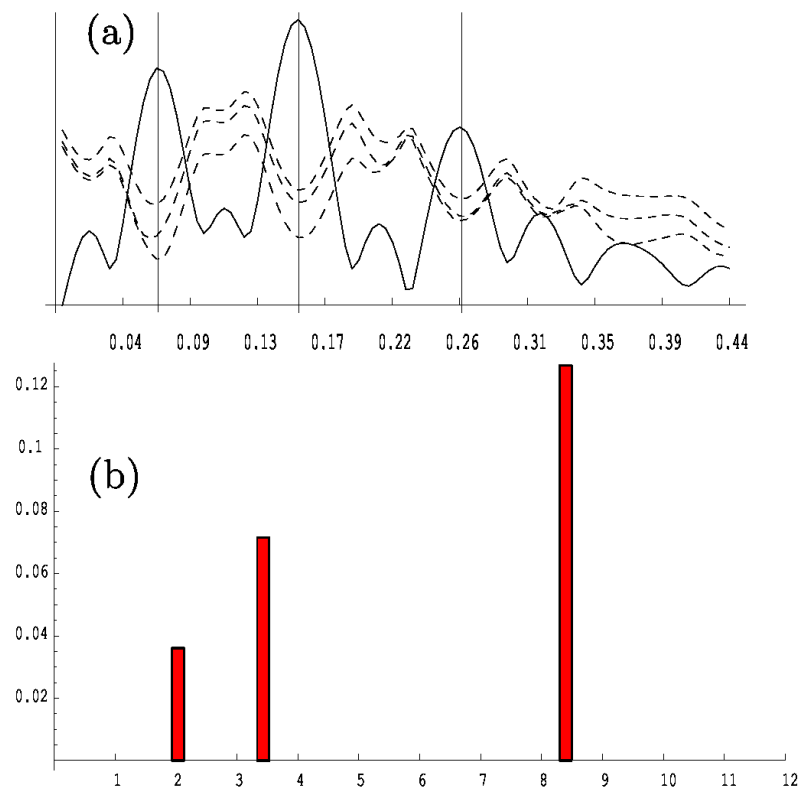

Fig. 4. Frequency identification (in the set $\Psi_{P, 1}$ ) and corresponding amplitude estimation: (a) the value of test statistics $\tilde{\Pi}_{n}(\{\psi\})=\sqrt{n}\left|\hat{r}_{n}(\psi)\right|$ (continuous line) and critical value $\tilde{c}_{n, b}^{\{\psi\}}(\alpha)$ (dashed line) for $\alpha \in$ $\{92 \%, 95 \%, 99 \%\}$ and $\psi$ from the set $\{(k-1) \pi / 720: k=$ $1,2, \ldots, 100\} ;$ (b) estimated amplitude corresponding to estimated frequencies from the set $\Psi_{P, 1}: X$ - estimated length of the cycle, $Y$ - estimated amplitude.

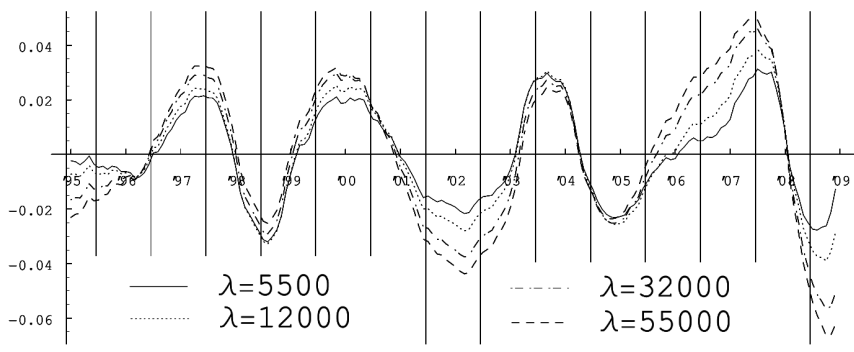

Fig. 5. Business cycle in industrial production after logarithm and application of centered moving average filter extracted by HP filter for $\lambda=5500$ (continuous line), $\lambda=12000$ (dotted line), $\lambda=32000$ (dotted and dashed line), $\lambda=55000$ (dashed line).

cycles with estimated length in the interval 3-4 years were supported in predominant set of subindices, only in the case of manufacture, food products and beverages (C10_C11), manufacture of basic pharmaceutical products and pharmaceutical preparations (C21) and electricity, gas, steam and air conditioning supply (D).

We see that the largest estimated amplitude characterizes cycles with the corresponding length of more than 4 years. But only in a few cases the cycles with length 5-8 years were supported. It can be seen clearly in Fig. 11, where the comparison of all identified cycles for all 32 indices is presented.
TABLE III

Categorised indices describing changes in economic activity in sectors and subsectors of industrial production in Poland.

B-D_F — Mining and quarrying; manufacturing; electricity, gas, steam and air conditioning supply; construction

MIG_ING_CAG - MIG Intermediate and capital goods

MIG_ING-MIG - MIG — Intermediate goods

MIG_CAG - Capital goods

MIG_DCOG - MIG - Durable consumer goods

MIG_NDCOG - MIG - Non-durable consumer goods

$\mathrm{B}-$ Mining and quarrying

$\mathrm{C}$ - Manufacturing

C10-C12 - Manufacture of food products; beverages and tobacco products

C10_C11 - Manufacture of food products and beverages

C10 - Manufacture of food products

C11 - Manufacture of beverages

C12 - Manufacture of tobacco products

C13_C14 - Manufacture of textiles and wearing apparel

$\mathrm{C} 15$ - Manufacture of leather and related products

C16 - Manufacture of wood and of products of wood and cork, except furniture; manufacture of articles of straw and plaiting materials

C17 - Manufacture of paper and paper products

C18 - Printing and reproduction of recorded media

C19 - Manufacture of coke and refined petroleum products

C20 - Manufacture of chemicals and chemical products

C21 - Manufacture of basic pharmaceutical products and pharmaceutical preparations

C22 - Manufacture of rubber and plastic products

C23 - Manufacture of other non-metallic mineral products

C24 - Manufacture of basic metals

C25 - Manufacture of fabricated metal products, except machinery and equipment

C26 - Manufacture of computer, electronic and optical products

C27 - Manufacture of electrical equipment

C28 - Manufacture of machinery and equipment ncan n.e.c.

C29 - Manufacture of motor vehicles, trailers and semitrailers

C29 C30 - Manufacture of motor vehicles, trailers, semi-trailers and of other transport equipment

C31 - Manufacture of furniture; other manufacturing

D - Electricity, gas, steam and air conditioning supply

In spite of the fact that observed time series were subject to filtering with the use of centered moving average filter $2 \times 12 \mathrm{MA}$, all investigated subindices provide data support in favour of the existence of cycles with length not greater than two years. However, as seen in Fig. 11, those short cycles are characterized by amplitudes with values located relatively close to zero, as compared with longer significant cycles. This makes such a short term periodic pattern not extremely important in describing cyclical behaviour of modelled time series. Additionally, all indices support cycles of length 3-4 years, with rela- 


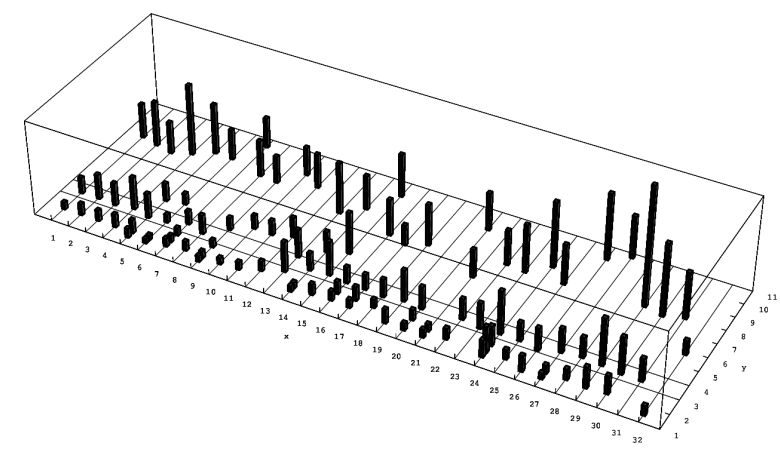

Fig. 11. Length of the cycles and amplitudes comparison: $X$ - the index number, $Y$ - estimated length of the cycle, $Z$ - estimated amplitude.

tively greater value of corresponding amplitudes as compared to characteristics of short term fluctuations. Also, except manufacture of wearing apparel (14th index) we see no data support for cycles with length between 4 and 7 years. Consequently, for all considered subindices, the set of statistically significant cycles is clearly divided in two separate parts. The first set is constituted by short term cycles with small amplitudes together with middle term fluctuations, attributed in most cases by stronger amplitudes. The second set consists of frequencies, describing long term cycle, namely with length not less than 7 years. Just like in case of the total production index, we tend not to interpret those long term fluctuations as important characteristic of business cycle for Polish economy. According to our results, just like for the total index, all considered subindices are characterized by existence of the long term trend.

Using HP filter we extract business cycles from all industrial production indexes. Similar as for industrial production index - total we fix the parameter $\lambda$ as $\lambda=5500, \lambda=12000, \lambda=32000, \lambda=55000$ (see Fig. 12). The reasons why we chose those values of $\lambda$ parameter are the following. Firstly, we fix the same parameter to compare results with those obtained for industrial production index. Secondly, the length of the cycle that is greater than 8 years is not clearly constant over different subindices and therefore we cannot interpret those fluctuations as business fluctuations. Finally, we can notice that in the interval from 4 to 8 years there are only a few significant lengths of cycles and this should give rise to extract similar shape of business fluctuations for different values of parameter $\lambda$. Almost all extracted fluctuations reveal presence of cycles with length in the interval 3-4 years. Summing up, the cycle with length in the range 3-4 years is typical and prevalent for cyclical fluctuations in industrial production in Poland.

\section{Concluding remarks}

In this paper a novel approach in business fluctuations analysis for one-dimensional economic processes is proposed. Using theory of almost periodically correlated time series and subsampling procedure we consider a formal approach to estimate the length of business cycles. The main advantage of our approach is that the business cycle characteristics are treated in formal way, and are subject to statistical inference. This clearly distinguishes presented framework from many filtering-based approaches, broadly considered in empirical applications. We model business fluctuations by parameters of discrete spectra of time series, under assumption that amplitude of these fluctuations is constant over time. Taking in consideration estimated length of the cycles we extract business fluctuations by HP filter for parameter of smoothness chosen on the basis of formal procedure.

The main conclusion presented in empirical illustration is that, during period 1995-2009, we confirm (using statistical tools) the presence of 3-4 years length of business cycle in industrial production index in Poland. This result was obtained either on the basis of the total index and also analysing subindices. This result confirms analyses conducted so far on the basis of Polish macroeconomic time series; see [46-49].

All indices and subindices supported significance of short term and middle term fluctuations, attaching relatively small amplitudes for periodicity with length less than 2 years. Additionally, in all time series we detected existence of longer term cycle ( $7-8$ years), interpreted in this paper as a trend or a mixture of both trend and business cycle fluctuations.

\section{Appendix}

Assumption 8.1. Let $\left\{X_{t}: t \in \mathbb{Z}\right\}$ be APC time series such that for any $x \in[0,2 \pi)$ there exists a constant $B(x)$ (which depends only on $x$ ), such that we have estimation

$$
\sum_{\psi \in \Psi \backslash\{x\}}\left|m(\psi) \operatorname{cosec}\left(\frac{\psi-x}{2}\right)\right|<B(x)<\infty .
$$

Theorem 8.1. Let $\left\{X_{t}: t \in \mathbb{N}\right\}$ be a time series for which the expectation function exists and it is almost periodic function of the form $\mu_{X}(t)=E\left(X_{t}\right)=$ $\sum_{\psi \in \Psi_{X}} m_{X}(\psi) \mathrm{e}^{\mathrm{i} \psi t}$. We assume that for the set $\Psi$ and corresponding Fourier coefficients $m(\cdot)$ Assumption 8.1 holds. Let $L(B)=\sum_{j=-p}^{q} a_{j} B^{j}$ be a linear filter, where $p, q \geq 0,\left\{a_{j}\right\}_{j=-p}^{q}$ is a sequence of real numbers, and $B^{j} X_{t}=X_{t-j}$ for any $j \in \mathbb{Z}$. Then

$$
E\left(Y_{t}\right)=\mu_{Y}(t)=\sum_{\psi \in \Psi_{Y}} m_{Y}(\psi) \mathrm{e}^{\mathrm{i} \psi t},
$$

where $\Psi_{Y}=\Psi_{X}$ and $m_{Y}(\psi)=L\left(\mathrm{e}^{-\mathrm{i} \psi}\right) m_{X}(\psi)$. Additionally, Assumption 8.1 holds for the set $\Psi_{Y}$ and corresponding coefficients $m_{Y}(\cdot)$.

Proof. Notice that

$$
\begin{gathered}
E\left(Y_{t}\right)=E\left(\sum_{j=p_{1}}^{p_{2}} a_{j} B^{j} X_{t}\right)=E\left(\sum_{j=p_{1}}^{p_{2}} a_{j} X_{t-j}\right) \\
=\sum_{j=p_{1}}^{p_{2}} a_{j} \sum_{\psi \in \Psi_{X}} m_{X}(\psi) \mathrm{e}^{\mathrm{i} \psi(t-j)}
\end{gathered}
$$




$$
\begin{aligned}
& =\sum_{\psi \in \Psi_{X}} m_{X}(\psi) \sum_{j=p_{1}}^{p_{2}} a_{j} \mathrm{e}^{-\mathrm{i} \psi j} \mathrm{e}^{\mathrm{i} \psi t} \\
& =\sum_{\psi \in \Psi_{X}} m_{X}(\psi) L\left(\mathrm{e}^{-\mathrm{i} \psi}\right) \mathrm{e}^{\mathrm{i} \psi t} .
\end{aligned}
$$

By estimation $\left|m_{Y}(\psi)\right| \leq\left|m_{X}(\psi)\right| \sum_{j=p_{1}}^{p_{2}}\left|a_{j}\right|$ we conclude that condition 1.1 from [24] holds for the set $\Psi_{Y}$ and corresponding Fourier coefficients $m_{Y}(\cdot)$.

Theorem 8.2. Take any $\psi \in(0,2 \pi)$. Let the assumptions of Theorem 2.2 in [24] hold. Then

(i) $\tilde{L}_{n, b}^{\{\psi\}}(x) \stackrel{p}{\rightarrow} J^{\{\psi\}}(x)$, for any $x \in \mathbb{R}$,

(ii) $\sup _{x \in \mathbb{R}}\left|\tilde{L}_{n, b}^{\{\psi\}}(x)-J^{\{\psi\}}(x)\right| \stackrel{p}{\longrightarrow} 0$,

(iii) subsampling confidence intervals for the parameter $|m(\psi)|$ are asymptotically consistent, which means that

$$
\begin{aligned}
& P\left(\sqrt{n}\left(\left|\hat{r}_{n}(\psi)\right|-|m(\psi)|\right) \leq \tilde{c}_{n, b}^{\{\psi\}}(1-\alpha)\right) \\
& \quad \longrightarrow 1-\alpha,
\end{aligned}
$$

where $b=b(n) \rightarrow \infty$ and $b / n \rightarrow 0$.

Proof of Theorem 8.2. In this proof we use the same steps as in Theorem 4.2.1, page 103 in [30]. Let $q=$ $n-b+1, \tau_{n}=\sqrt{n}$ and

$$
\begin{aligned}
& U_{n}(x) \\
& \quad=\frac{1}{q} \sum_{t=1}^{q \mathbf{1}}\left\{\tau_{b}\left(\left|\hat{m}_{n}^{t-1, b}(\psi)\right|-|m(\psi)|\right) \leq x\right\} .
\end{aligned}
$$

Notice that

$$
\begin{gathered}
\tilde{L}_{n, b}^{\{\psi\}}(x)=\frac{1}{q} \sum_{t=1}^{q \mathbf{1}}\left\{\tau_{b}\left[\left|\hat{m}_{n}^{t-1, b}(\psi)\right|-|m(\psi)|\right]\right. \\
+\tau_{b}\left[\left(|m(\psi)|-\left|\hat{r}_{n}(\psi)\right|\right)+\left(\left|\hat{r}_{n}^{t-1, b}(\psi)\right|\right.\right. \\
\left.\left.\left.\quad-\left|\hat{m}_{n}^{t-1, b}(\psi)\right|\right)\right] \leq x\right\} .
\end{gathered}
$$

We need the following lemma.

Lemma 8.1. For any real $x$ and $\epsilon>0$ we have estimation

$$
\begin{aligned}
& U_{n}(x-\epsilon) \mathbf{1}\left\{E_{n}\right\} \leq \tilde{L}_{n, b}^{\{\psi\}}(x) \mathbf{1}\left\{E_{n}\right\} \\
& \quad \leq U_{n}(x+\epsilon),
\end{aligned}
$$

where $E_{n}=\left\{\tau_{b} \max _{1 \leq t \leq q} \mid\left(|m(\psi)|-\left|\hat{r}_{n}(\psi)\right|\right)+\right.$ $\left.\left(\left|\hat{r}_{n}^{t-1, b}(\psi)\right|-\left|\hat{m}_{n}^{t-1, b}(\psi)\right|\right) \mid \leq \bar{\epsilon}\right\}$.

Proof. Let consider two cases:

$1^{\circ} \mathbf{1}\left\{E_{n}\right\}=0$, inequality (27) holds.

$2^{\circ} \mathbf{1}\left\{E_{n}\right\}=1$, then

$$
\begin{aligned}
& \tau_{b} \max _{1 \leq t \leq q} \mid\left(|m(\psi)|-\left|\hat{r}_{n}(\psi)\right|\right) \\
& \quad+\left(\left|\hat{r}_{n}^{t-1, b}(\psi)\right|-\left|\hat{m}_{n}^{t-1, b}(\psi)\right|\right) \mid \leq \epsilon,
\end{aligned}
$$

which means that for any $1 \leq t \leq q$

$$
\begin{aligned}
& \eta_{n}^{t-1, b}(\psi):=\tau_{b}\left[\left(|m(\psi)|-\left|\hat{r}_{n}(\psi)\right|\right)\right. \\
& \left.\quad+\left(\left|\hat{r}_{n}^{t-1, b}(\psi)\right|-\left|\hat{m}_{n}^{t-1, b}(\psi)\right|\right)\right] \in[-\epsilon, \epsilon] .
\end{aligned}
$$

Using next inequality $x-\eta_{n}^{t-1, b}(\psi) \geq x-\epsilon$, which is true for any $1 \leq t \leq q$ we get

$$
\mathbf{1}\left\{\tau_{b}\left[\left|\hat{m}_{n}^{t-1, b}(\psi)\right|-|m(\psi)|\right]\right.
$$

$$
\begin{aligned}
& +\tau_{b}\left[\left(|m(\psi)|-\left|\hat{r}_{n}(\psi)\right|\right)\right. \\
& \left.\left.+\left(\left|\hat{r}_{n}^{t-1, b}(\psi)\right|-\left|\hat{m}_{n}^{t-1, b}(\psi)\right|\right)\right] \leq x\right\} \\
& =\mathbf{1}\left\{\tau_{b}\left[\left|\hat{m}_{n}^{t-1, b}(\psi)\right|-|m(\psi)|\right]\right. \\
& \left.\leq x-\eta_{n}^{t-1, b}(\psi)\right\} \\
& \geq \mathbf{1}\left\{\tau_{b}\left[\left|\hat{m}_{n}^{t-1, b}(\psi)\right|-|m(\psi)|\right] \leq x-\epsilon\right\} .
\end{aligned}
$$

Analogically, using inequality $x-\eta_{n}^{t-1, b}(\psi) \leq x+\epsilon$ we get

$$
\begin{aligned}
\mathbf{1}\left\{\tau_{b}\right. & {\left[\left|\hat{m}_{n}^{t-1, b}(\psi)\right|-|m(\psi)|\right] } \\
& +\tau_{b}\left[\left(|m(\psi)|-\left|\hat{r}_{n}(\psi)\right|\right)\right. \\
& \left.\left.+\left(\left|\hat{r}_{n}^{t-1, b}(\psi)\right|-\left|\hat{m}_{n}^{t-1, b}(\psi)\right|\right)\right] \leq x\right\} \\
& =\mathbf{1}\left\{\tau_{b}\left[\left|\hat{m}_{n}^{t-1, b}(\psi)\right|-|m(\psi)|\right]\right. \\
& \left.\leq x-\eta_{n}^{t-1, b}(\psi)\right\} \\
& \leq \mathbf{1}\left\{\tau_{b}\left[\left|\hat{m}_{n}^{t-1, b}(\psi)\right|-|m(\psi)|\right] \leq x+\epsilon\right\} .
\end{aligned}
$$

Summing inequality (28), (29) for $t=1,2, \ldots, q$ we get (27). This completes the proof of lemma.

In next step we show that $P\left(E_{n}\right) \rightarrow 0$. Using inequality ||$z_{1}|-| z_{2}|| \leq\left|z_{1}-z_{2}\right|$ (which is true for any complex numbers $\left.z_{1}, z_{2}\right)$ and inequality $\left|\sum_{j=p}^{q} c_{j} \mathrm{e}^{\mathrm{i} j x}\right| \leq$ $c_{p}|\operatorname{cosec}(x / 2)|$ (which is true for any $x \not \equiv 0$ modulo $2 \pi$ and real numbers $c_{p} \geq c_{p+1} \geq \ldots \geq c_{q}$ ) we have

$$
\begin{aligned}
& \max _{1 \leq t \leq q}\left|\eta_{n}^{t-1, b}(\psi)\right| \leq \max _{1 \leq t \leq q} \tau_{b}|| m(\psi)|-| \hat{r}_{n}(\psi)|| \\
& \quad+\max _{1 \leq t \leq q} \tau_{b}|| \hat{r}_{n}^{t-1, b}(\psi)|-| \hat{m}_{n}^{t-1, b}(\psi)|| \\
& \quad \leq \tau_{b}|| m(\psi)|-| \hat{r}_{n}(\psi)|| \\
& \quad+\max _{1 \leq t \leq q} \tau_{b}\left|\hat{r}_{n}^{t-1, b}(\psi)-\hat{m}_{n}^{t-1, b}(\psi)\right| \\
& \quad \leq \tau_{b}\left|m(\psi)-\hat{r}_{n}(\psi)\right| \\
& \quad+\frac{\tau_{b}}{b}\left|\overline{\boldsymbol{X}}_{n}\right||\operatorname{cosec}(\psi / 2)| \\
& \quad \leq \tau_{b}\left|m(\psi)-\hat{m}_{n}(\psi)\right| \\
& \quad+\tau_{b}\left|\hat{m}_{n}(\psi)-\hat{r}_{n}(\psi)\right|+\frac{\tau_{b}}{b}\left|\overline{\boldsymbol{X}}_{n}\right||\operatorname{cosec}(\psi / 2)| \\
& \quad \leq \tau_{b}\left|m(\psi)-\hat{m}_{n}(\psi)\right|+\frac{\tau_{b}}{n}\left|\overline{\boldsymbol{X}}_{n}\right||\operatorname{cosec}(\psi / 2)| \\
& \quad+\frac{\tau_{b}}{b}\left|\overline{\boldsymbol{X}}_{n}\right||\operatorname{cosec}(\psi / 2)| .
\end{aligned}
$$

By convergence $\tau_{b}\left|m(\psi)-\hat{m}_{n}(\psi)\right| \stackrel{p}{\rightarrow} 0$ and $\frac{\tau_{b}}{b}\left|\overline{\boldsymbol{X}}_{n}\right| \stackrel{p}{\rightarrow} 0$ we get

$$
\max _{1 \leq t \leq q}\left|\eta_{n}^{t-1, b}(\psi)\right| \stackrel{p}{\rightarrow} 0,
$$

which means that $P\left(E_{n}\right) \rightarrow 1$. Using next Slutsky's Lemma and Theorem 2.1 in [24] we have $\sqrt{n}\left(\left|r_{n}^{t-1, b}(\psi)\right|-\right.$ $|m(\psi)|) \stackrel{d}{\rightarrow} J^{\{\psi\}}$. To finish the proof it is sufficient to follow next steps in Theorem 4.2.1, page 103 in [30], therefore we omit them. 


\section{Acknowledgments}

The authors would like to thank Paweł Skrzypczyński, Michał Gradzewicz, Jakub Growiec, Andrzej Kocięcki and Michał Rubaszek for many valuable suggestions and remarks.

\section{References}

[1] W.C. Mitchell, Business cycles as revealed by business annals, in: Business Annals, Ed. W.L. Thorp, 1926.

[2] R.E. Lucas, Understanding business cycles, in: Stabilization of the Domestic and International Economy, Eds. K. Brunner, A.H. Meltzer, Carnegie, Rochester Conference Series on Public Policy, no. 5, reprinted in Kydland, Ed. E. Finn, Business Cycle Theory, E.E. Aldershot, 1977, p. 85.

[3] R.G. King, S.T. Rebelo, Resusticating real business cycles, in: Handbook of Macroeconomics, Eds. J.B. Taylor, M. Woodford, Elsevier, London 1999, p. 927.

[4] J.H. Stock, M.W. Watson, Business cycle fluctuations in US macroeconomic time series, in: Handbook of Macroeconomics, Eds. J.B. Taylor, M. Woodford, Elsevier, London 1999, p. 3.

[5] F.X. Diebold, G. Rudenbush, Rev. Econom. Statist. 78, 67 (1996).

[6] E.G. Gladyshev, Sov. Math. 2, 995 (1961).

[7] E. Parzen, M. Pagano, J. Econometrics 9, 137 (1979).

[8] D.R. Osborn, J.P. Smith, J. Busin. Econom. Statist. 9, 117 (1989).

[9] P.H. Franses, H.P. Boswijk, Statist. Probabil. Lett. 30, 235 (1996).

[10] P.H. Franses, Stochastic Trends in Economic Time Series, Oxford University Press, New York 1996.

[11] T. Bollerslev, E. Ghysels, J. Busin. Economic Statist. 14, 139 (1996).

[12] P.H. Franses, M. Ooms, Int. J. Forecast. 13, 117 (1997).

[13] P. Burridge, A.M. Taylor, J. Econometr. 104, 91117 (2001).

[14] P.H. Franses, D. Dijk, Int. J. Forecast. 21, 87 (2005).

[15] H.L. Hurd, A.G. Miamee, Periodically Correlated Random Sequences: Spectral Theory and Practice, Wiley, Hoboken 2007.

[16] C. Corduneanu, Almost Periodic Functions, Chelsea, New York 1989.

[17] W.A. Gardner, Signal Proc. 34, 1111 (1986).

[18] A. Napolitano, C.M. Spooner, IEEE Trans. Signal Proc. 49, 30 (2001).

[19] P. Bloomfield, H. Hurd, R. Lund, J. Time Ser. Anal. 15, 127 (1994).

[20] W.A. Gardner, A. Napolitano, L. Paura, Signal Proc. 86, 639 (2006).

[21] E. Serpedin, F. Panduru, I. Sari, G.B. Giannakis, Signal Proc. 85, 2233 (2005).

[22] H. Hurd, J. Multivariate Anal. 37, 24 (1991).

[23] M.B. Priestley, Spectral Analysis and Time Series, Academic Press, London 1981.
[24] Ł. Lenart, J. Multivariate Anal. 115, 252 (2013).

[25] H. Hurd, J. Multivariate Anal. 29, 53 (1989).

[26] D. Dehay, H. Hurd, in: Cyclostationarity in Communications and Signal Processing, Ed. W.A. Gardner, IEEE Press, 1994, p. 295.

[27] Ł. Lenart, Bernoulli 17, 290 (2011).

[28] Ł. Lenart, J. Leśkow, R. Synowiecki, J. Time Ser. Anal. 29, 995 (2008).

[29] A.M. Walker, Biometrika 58, 21 (1971).

[30] D. Politis, J. Romano, M. Wolf, Subsampling, Springer-Verlag, New York 1999.

[31] S. Beveridge, C.R. Nelson, J. Monet. Econom. 7, 151 (1981).

[32] P.K. Clark, Quart. J. Econom. 102, 797 (1987).

[33] A.C. Harvey, A. Jaeger, J. Appl. Econometr. 8, 231 (1993).

[34] J.D. Hamilton, Econometrica 57, 357 (1989).

[35] H.-M. Krolzig, Markov-Switching Vector Autoregressions, Modelling, Statistical Inference and Application to Business Cycle Analysis, Springer-Verlag, Berlin 1997.

[36] S. Makridakis, S.C. Wheelwright, R.J. Hyndman, Forecasting: Methods and Applications, Wiley, New York 1998.

[37] P.J. Brockwell, R.A. Davis, Introduction to Time Series and Forecasting, Springer-Verlag, New York 2002.

[38] F. Canova, J. Monet. Econom. 41, 475 (1998).

[39] C. Burnside, J. Monet. Econom. 41, 513 (1998).

[40] R.J. Hodrick, E.C. Prescott, J. Money, Credit Bank. 29, 1 (1997).

[41] V. Gómez, J. Busin. Econom. Statist. 17, 109 (1999).

[42] V. Gómez, J. Busin. Econom. Statist. 19, 365 (2001).

[43] A. Maravall, Ana del Río, Time aggregation and the Hodrick-Prescott filter, Banco de España - Servicio de Estudios Documento de Trabajo no. 0108, 2001.

[44] J.R. Hicks, A Contribution to the Theory of the Trade Cycle, Clarendon Press, Oxford 1950.

[45] C. Milas, P.A. Rothman, D. Dijk, Nonlinear Time Series Analysis of Business Cycles, Elsevier, Amsterdam 2006.

[46] M. Gradzewicz, J. Growiec, J. Hagemejer, P. Popowski, Bank $i$ Kredyt 41, 41 (2010) (in Polish).

[47] E. Adamowicz, S. Dudek, D. Pachucki, E. Walczyk, Business cycle synchronisation between Polish economy and Euro zone in the context of structure, Instytut Rozwoju Gospodarczego, Szkoła Główna Handlowa, Warszawa 2008 (in Polish).

[48] P. Skrzypczyński, Fluctuations of economic activity in Poland and Euro zone, Documents and Studies of National Bank of Poland, no. 227, Warsaw 2008, (in Polish).

[49] P. Skrzypczyński, Analysis of business cycle synchronisation in Euro zone, Documents and Studies of $\mathrm{Na}$ tional Bank of Poland, no. 210, Warsaw 2006, (in Polish). 

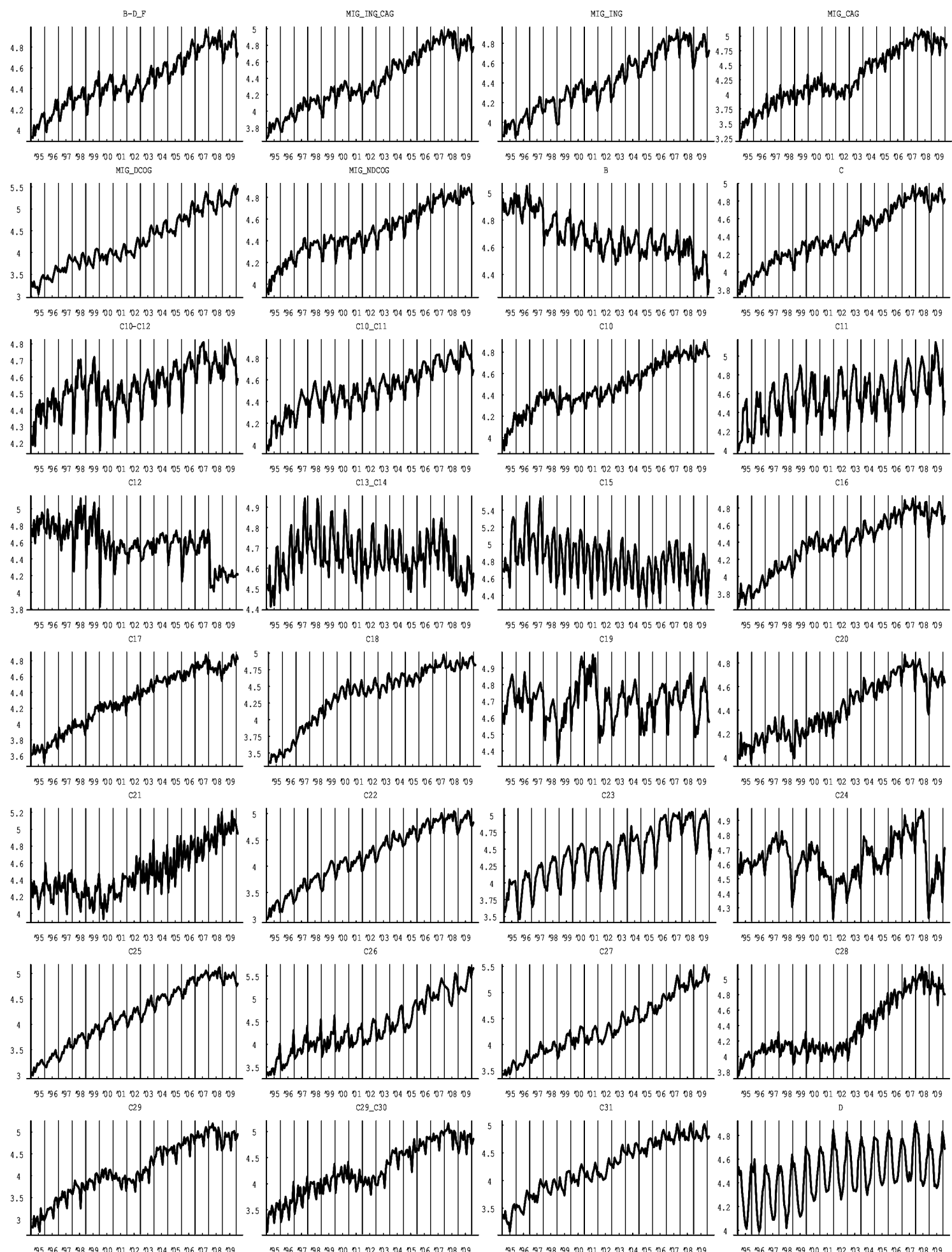

Fig. 6. Logarithm of industrial production indices in Poland $(2005$ year $=100 \%)$ in sectors and subsectors from January 1995 to February 2010. 

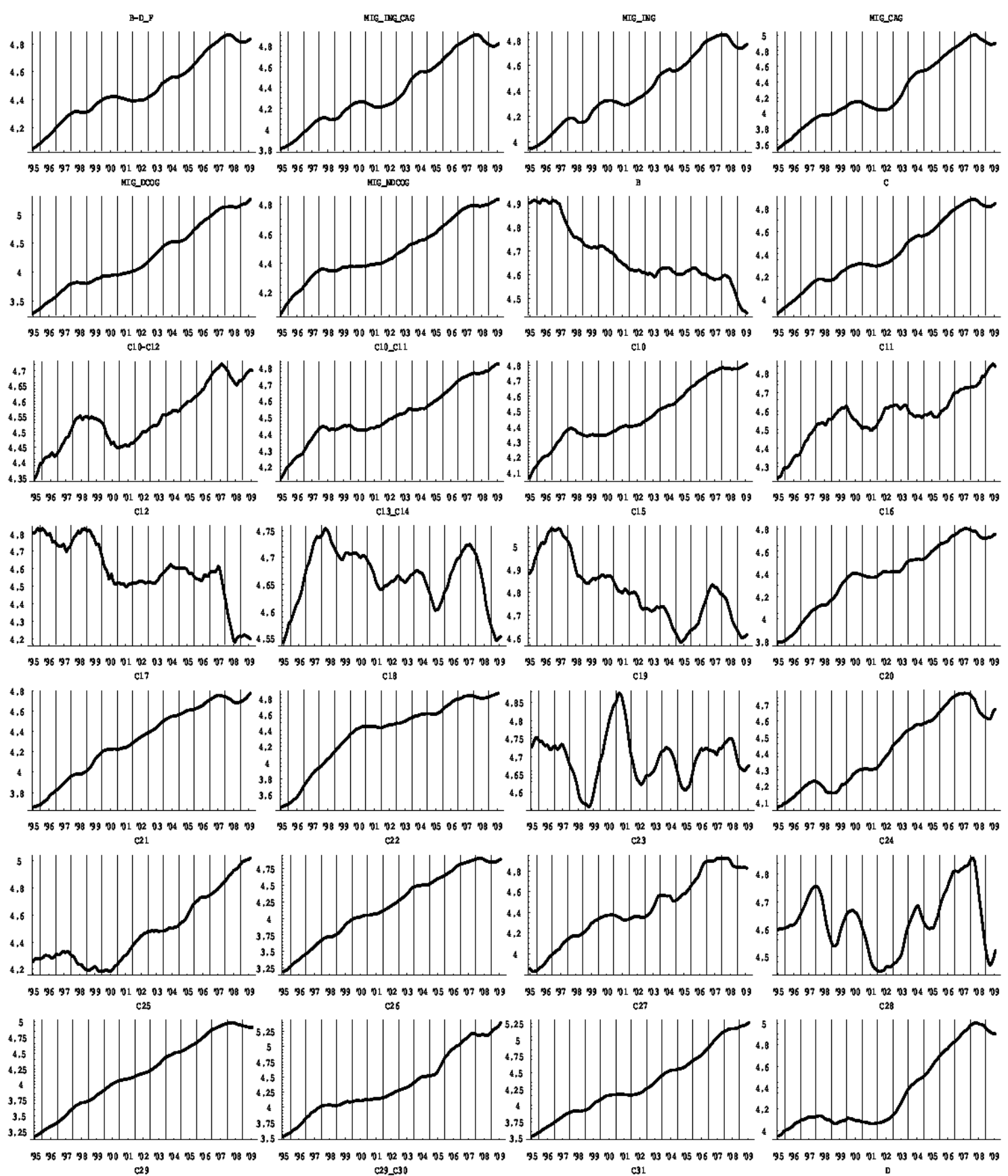

C26

c27

C28
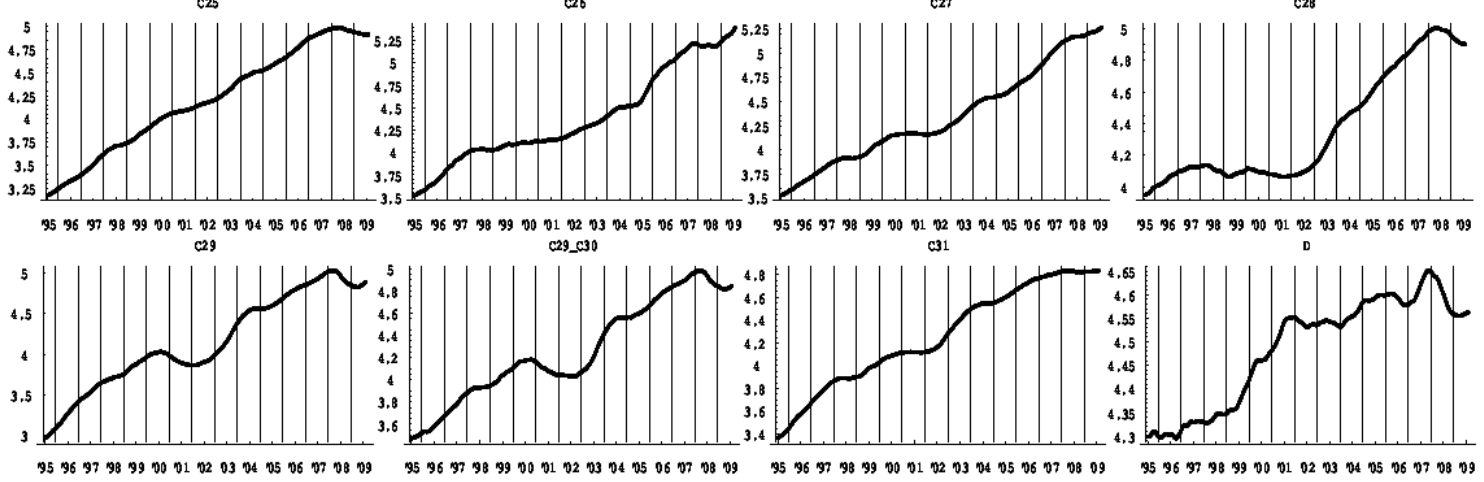

Fig. 7. Realizations of centered moving average filter $2 \times 12 \mathrm{MA}$ applied for logarithm of industrial production indexes in Poland in sectors and subsectors. 

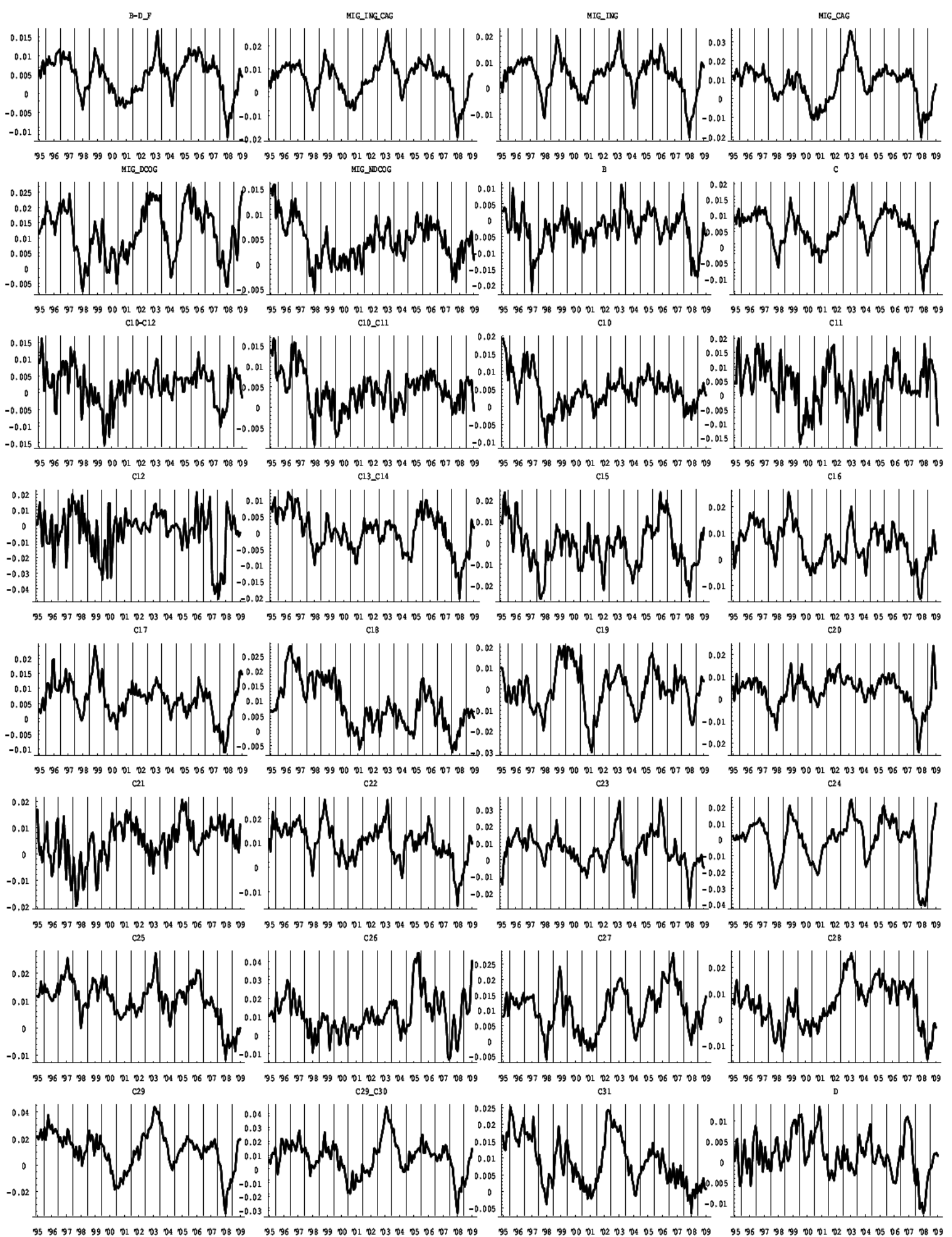

Fig. 8. First difference for realization of centered moving average filter $2 \times 12 \mathrm{MA}$ applied for logarithm of industrial production indexes in sectors and subsectors. 

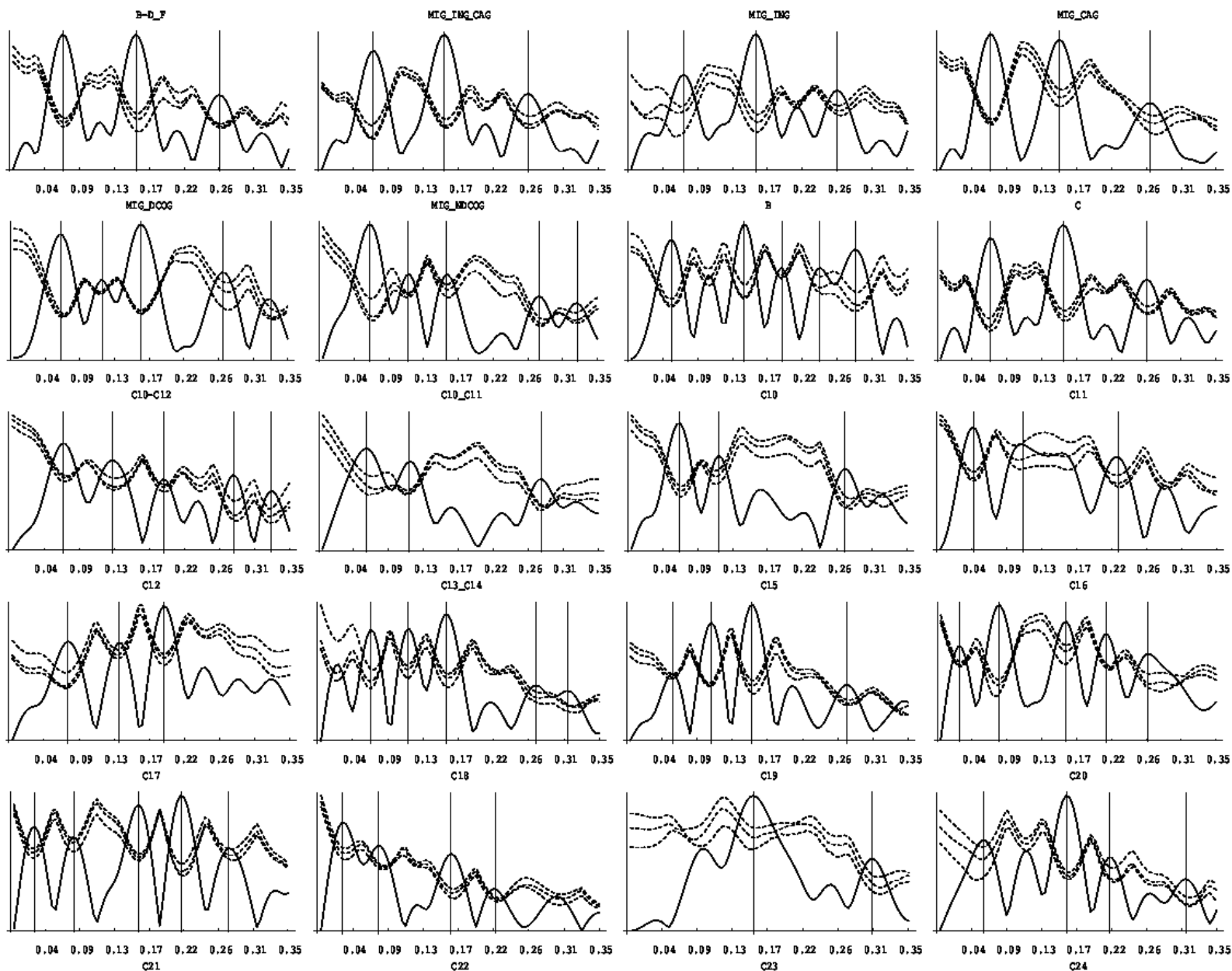

C19
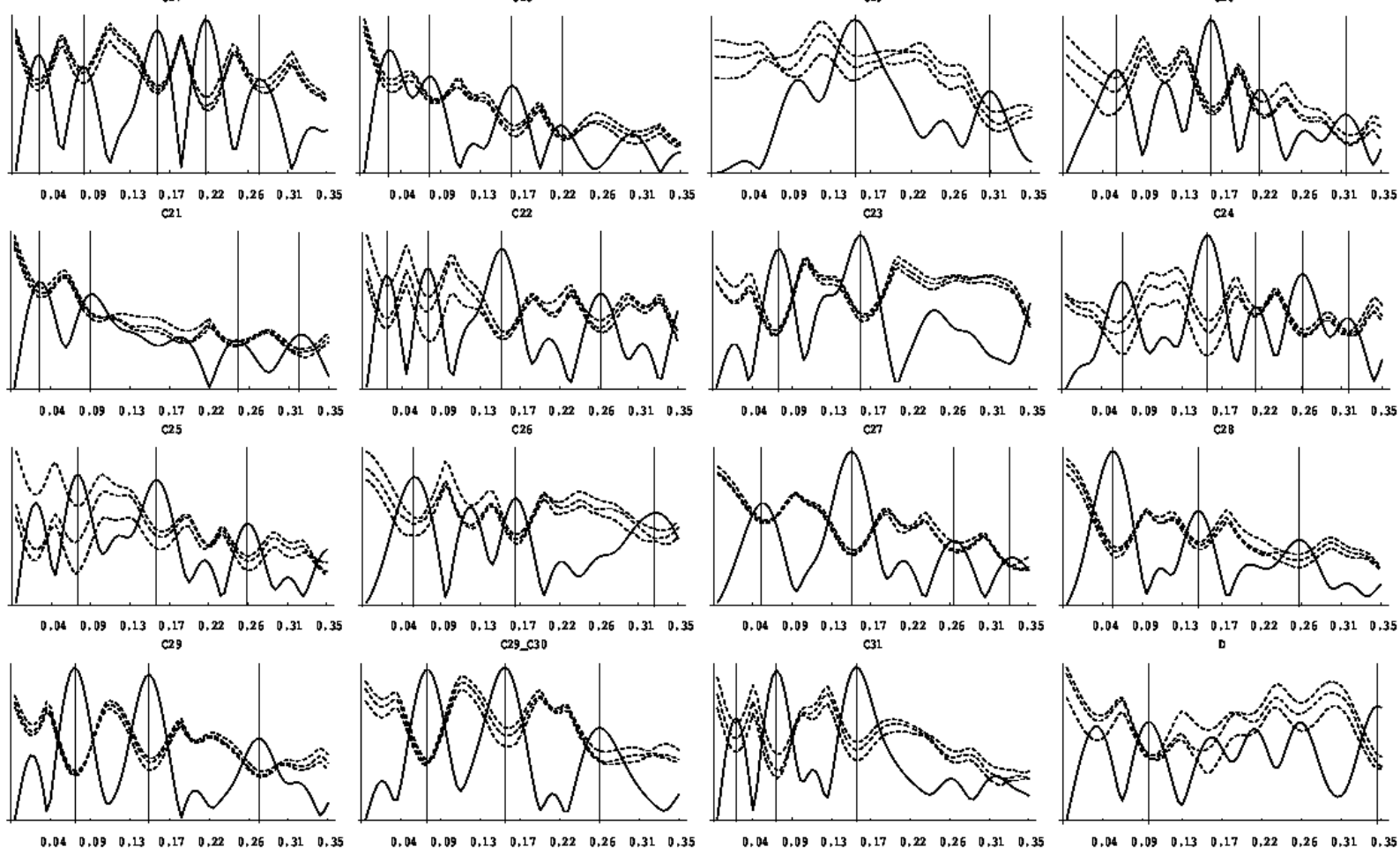

Fig. 9. Frequency identification (in the set $\left.\Psi_{P, 1}\right)$ : continuous line - the value of test statistics $\tilde{\Pi}_{n}(\{\psi\})=\sqrt{n}\left|\hat{r}_{n}(\psi)\right|$, dashed line - critical value $\tilde{c}_{n, b}^{\{\psi\}}(\alpha)$ for $\alpha \in\{92 \%, 95 \%, 99 \%\}$ and $\psi$ from the set $\{(k-1) \pi / 720: k=1,2, \ldots, 100\}$. 

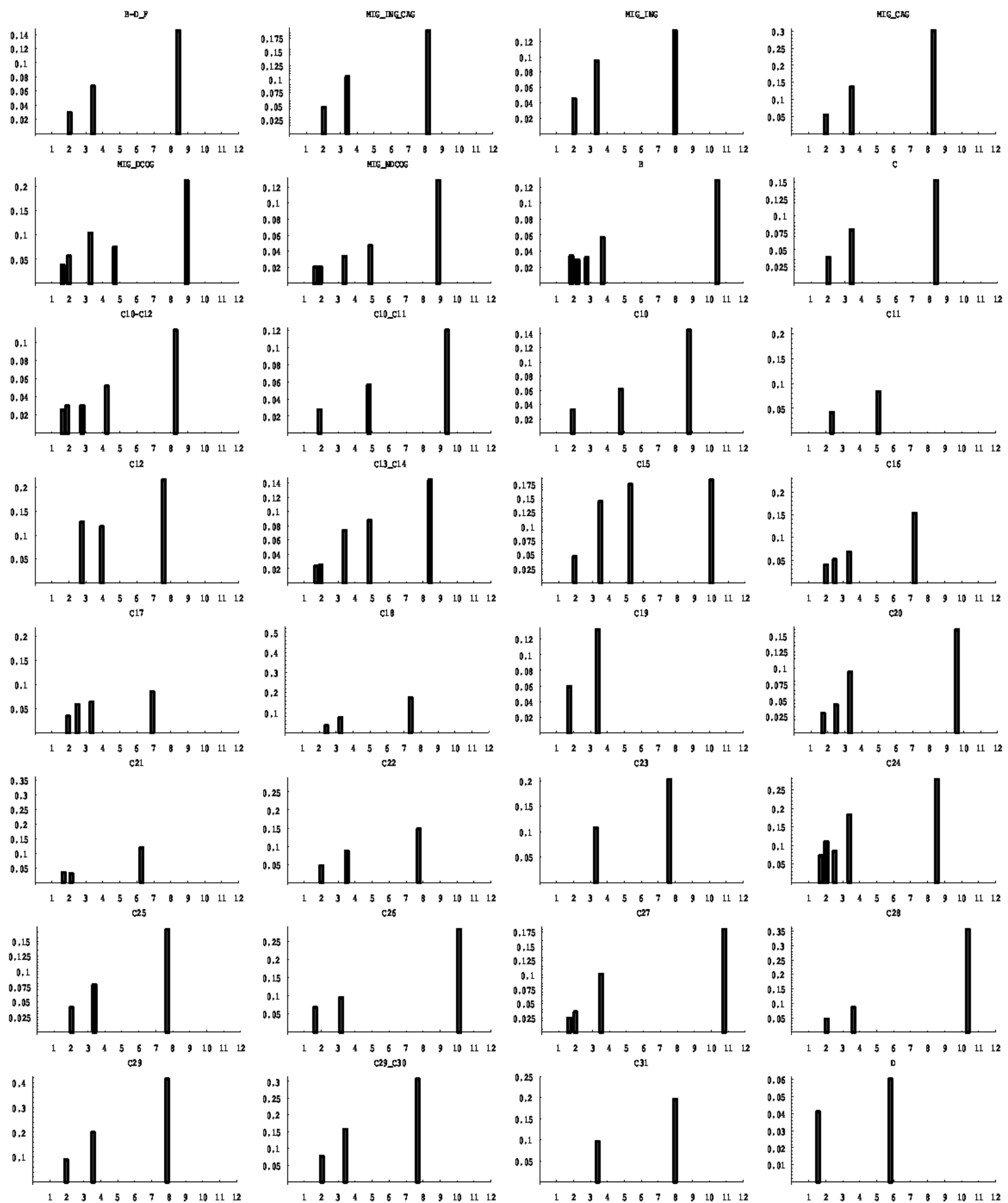

Fig. 10. Estimated amplitude and estimated length of the cycles connected with identified frequencies in the set $\Psi_{P, 1}$ : $X$ - estimated length of the cycle, $Y$ - estimated amplitude. 

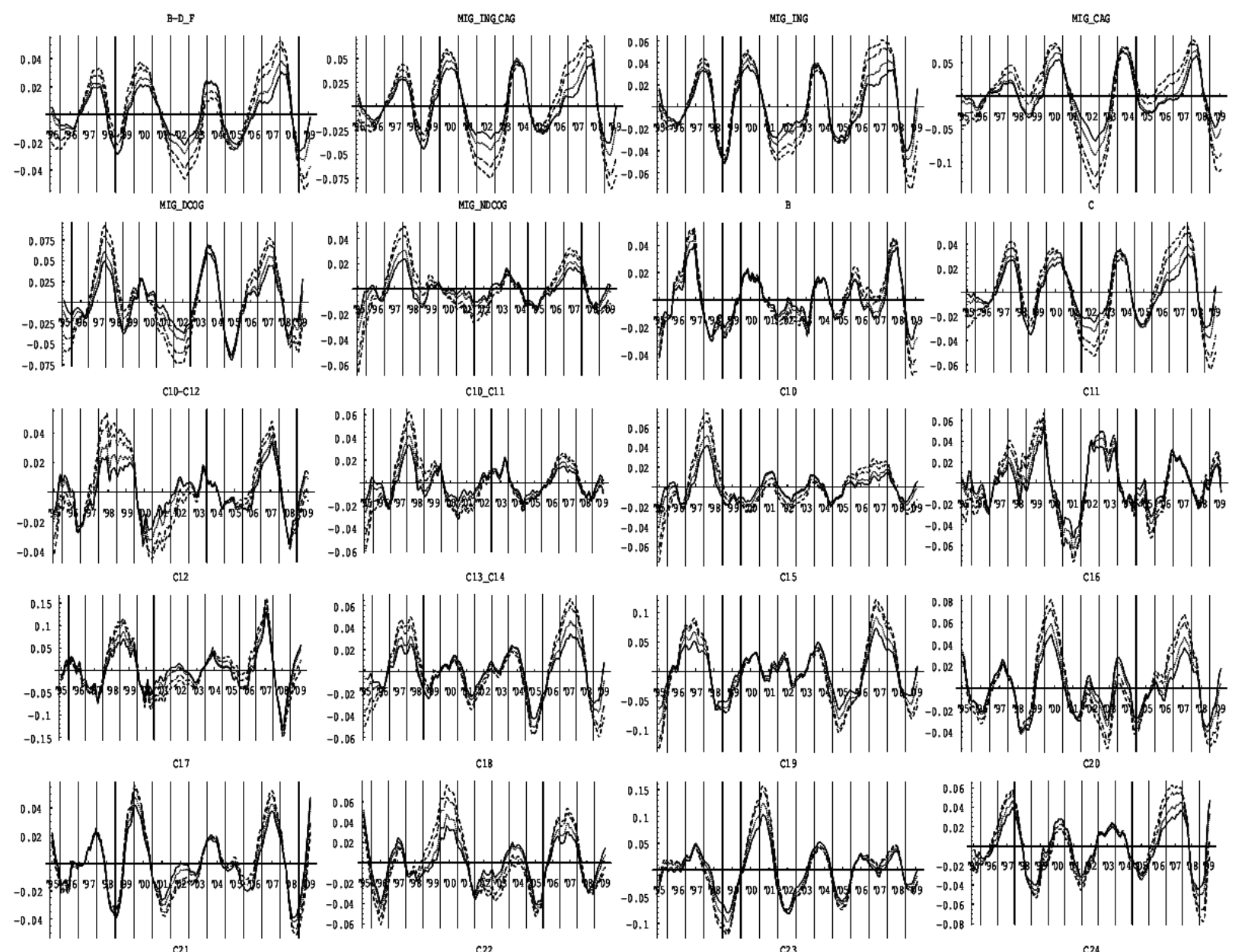

C18

019

C20
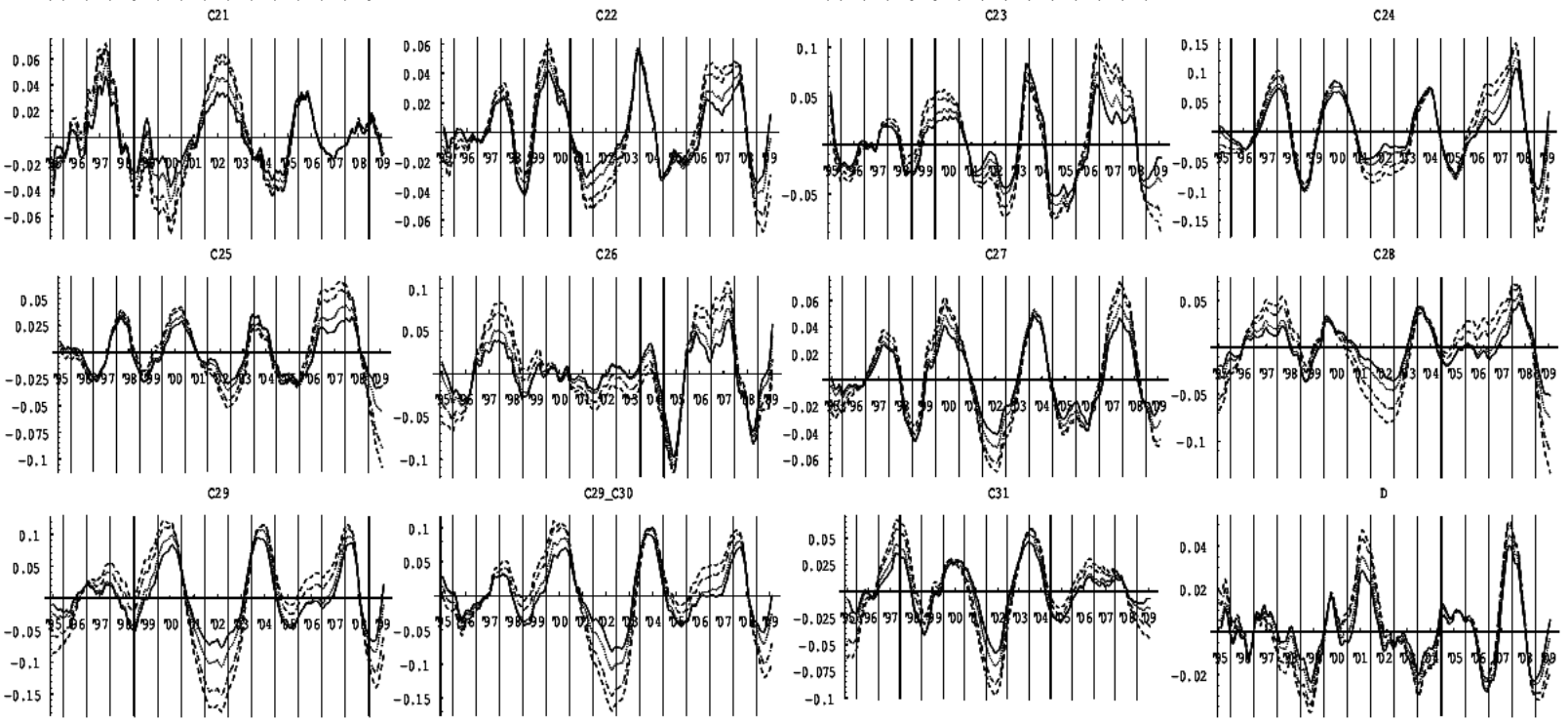

Fig. 12. Business cycle (extracted by HP filter) in sectors and subsectors of industrial production after logarithm and application of centered moving average filter for $\lambda=5500$ (continuous line), $\lambda=12000$ (dotted line), $\lambda=32000$ (doted and dashed line), $\lambda=55000$ (dashed line). 